Notre Dame Journal of Formal Logic

Volume 47, Number 4, 2006

\title{
Categorical Abstract Algebraic Logic: More on Protoalgebraicity
}

\author{
George Voutsadakis
}

\begin{abstract}
Protoalgebraic logics are characterized by the monotonicity of the Leibniz operator on their theory lattices and are at the lower end of the Leibniz hierarchy of abstract algebraic logic. They have been shown to be the most primitive among those logics with a strong enough algebraic character to be amenable to algebraic study techniques. Protoalgebraic $\pi$-institutions were introduced recently as an analog of protoalgebraic sentential logics with the goal of extending the Leibniz hierarchy from the sentential framework to the $\pi$-institution framework. Many properties of protoalgebraic logics, studied in the sentential logic framework by Blok and Pigozzi, Czelakowski, and Font and Jansana, among others, have already been adapted in previous work by the author to the categorical level. This work aims at further advancing that study by exploring in this new level some more properties of protoalgebraic sentential logics.
\end{abstract}

\section{Introduction}

This paper is a companion to [24], where the notion of an $N$-protoalgebraic $\pi$ institution was introduced. The inspiration and the motivation comes from the theory of protoalgebraic sentential logics which were introduced by Blok and Pigozzi in [3] and have been widely acknowledged to form the widest class of logics amenable to universal algebraic study techniques [12]. A short introduction to the topic will be presented here since the reader is advised to consult the Introduction of [24] for more details on the history of the topic and on the motivation for its study in the institution framework. For sentential logics, the best part of [8] is devoted to protoalgebraicity.

Received March 8, 2005; accepted March 26, 2006; printed December 28, 2006 2000 Mathematics Subject Classification: Primary, 03G99; Secondary, 18C15, 68N30

Keywords: algebraic logic, equivalent deductive systems, equivalent institutions, protoalgebraic logics, equivalential logics, algebraizable deductive systems, adjunctions, equivalent categories, algebraizable institutions, Leibniz operator, Tarski operator, Leibniz hierarchy

(c)2006 University of Notre Dame 
The Leibniz hierarchy of abstract algebraic logic categorizes logics into several classes that roughly reflect the extent to which a logic may be studied using universal algebraic techniques. The most important steps in this hierarchy are formed by the classes of protoalgebraic [3] (see also [7]), equivalential [6] (see also [20]), and algebraizable [4] (see also [16], [17], [18]) logics. The book by Czelakowski [8] and the survey article by Font, Jansana, and Pigozzi [12] provide overviews of the theory and of the Leibniz hierarchy. A schematic representation of the different main levels is given below, where an arrow represents an inclusion relation between two classes.

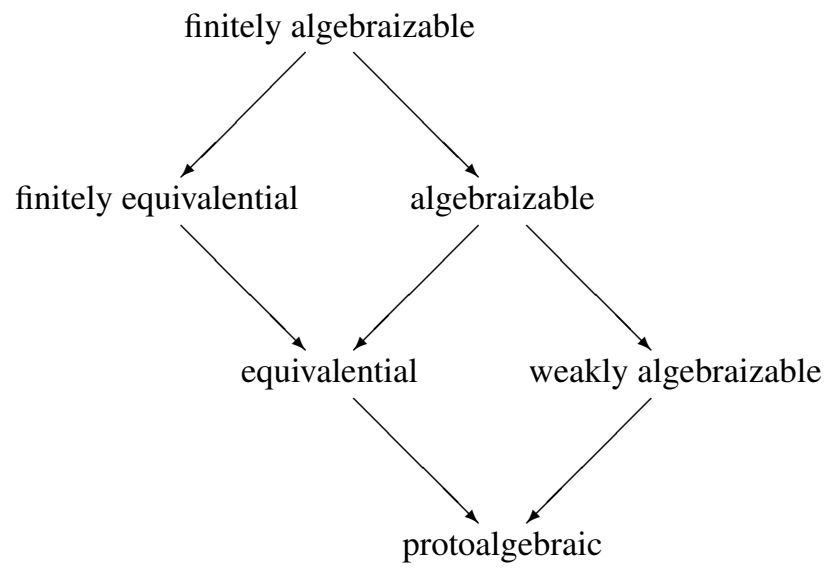

According to their original definition [3], a sentential logic $\delta=\left\langle\mathcal{L}, \vdash_{\S}\right\rangle$ is protoalgebraic if, for every theory $T$ of $\mathcal{S}$, and all $\mathcal{L}$-formulas $\varphi, \psi \in \operatorname{Fm}_{\mathcal{L}}(V)$,

$$
\langle\varphi, \psi\rangle \in \Omega(T) \text { implies } T, \varphi \vdash_{s} \psi ;
$$

that is, if two formulas are congruent modulo the Leibniz congruence $\Omega(T)$, then they are interderivable modulo the theory $T$, for every theory $T$ of $\$$. This property turns out to be equivalent to the monotonicity of the Leibniz operator on the theories of the logic. More precisely, $\&$ is protoalgebraic if and only if, for all theories $T_{1}, T_{2}$ of $\delta$,

$$
T_{1} \subseteq T_{2} \text { implies } \Omega\left(T_{1}\right) \subseteq \Omega\left(T_{2}\right) .
$$

Font and Jansana in [13] show that protoalgebraicity of a sentential logic $\&$ may also be characterized via the Tarski operator $\widetilde{\Omega}$ by the property that, for every theory $T$ of s,

$$
\widetilde{\Omega}\left(f^{T}\right)=\Omega(T),
$$

where $\delta^{T}=\left\langle\mathcal{L}, \vdash_{\delta^{T}}\right\rangle$ is defined, for every $\Phi \cup\{\varphi\} \subseteq \mathrm{Fm}_{\mathcal{L}}(V)$, by

$$
\Phi \vdash_{\delta^{T}} \varphi \quad \text { iff } T, \Phi \vdash_{\S} \varphi .
$$

This characterization has the important consequence that $\delta$ is protoalgebraic if and only if for any $\mathcal{L}$-algebra $\mathbf{A}$, every full model of $\delta$ over $\mathbf{A}$ consists of a principal filter of the lattice of all $\&$-filters on $\mathbf{A}$ (Theorem 3.4 of [13]).

In [24], the author proposed an extension of these results in order to cover the case of logical systems formalized as $\pi$-institutions [11] (see also [14], [15]). The introduction of protoalgebraic $\pi$-institutions was a culmination of a long-term project 
undertaken by the author with the goal of adapting general definitions, methods, and results widely applied to the theory of algebraizability of deductive systems to $\pi$ institutions. The first phase of this project, more syntactic in nature and influenced mainly by the works of Blok and Pigozzi ([2], [4]), appeared in [26] (see also [27], [28], [29]). A second phase, with a model-theoretic flavor, based on the introduction of a categorical Tarski operator and heavily influenced by the work of Font and Jansana and other members of the Barcelona algebraic logic group (see [13] for an overview and an extensive bibliography), led to the series of papers [21], [22], [23], [24], [25], [30], [31], which are precursors to the current work.

A summary of the contents of the present paper is now presented. A brief review of the basic definitions and properties concerning $N$-protoalgebraic $\pi$-institutions that were introduced and studied in [24] will be given in Section 2. In Section 3, implication systems will be studied in the $\pi$-institution framework. Paralleling the sentential logic setting, an implication system is a collection of natural transformations on the sentences of a $\pi$-institution that satisfies properties analogous to the reflexivity and the modus ponens of sentential implication systems [8]. The main result of the section is that the existence of an implication system implies protoalgebraicity of a $\pi$-institution, an analog of the easy direction (v) $\rightarrow$ (i) of Theorem 1.1.3 of [8]. The reverse implication, which is valid in the sentential logic framework, does not seem to be true, in general, in the $\pi$-institution framework. Along similar lines, it is also shown that a $\pi$-institution with a version of the parameterized local deduction-detachment theorem is also protoalgebraic. Again, for a comprehensive overview of the connections between protoalgebraicity and the deduction theorem in the sentential logic framework, the reader is advised to consult Chapter 2 of [8]. In Section 4, the study of parameterized equivalence systems is undertaken. Once more the relevant definition is an appropriate translation of the one for sentential logics (Definition 1.2.3 of [8]) with a slight complication due to the presence of signature morphisms in the $\pi$-institution setting. The development in [8] is followed closely and the main result here is that the existence of a parameterized equivalence system implies protoalgebraicity, an analog of the easy direction (ii) $\rightarrow$ (i) of Theorem 1.2.7 of [8]. In Section 5, we return to some more model-theoretic consequences of protoalgebraicity showing that a variety of results of [13] pertaining to protoalgebraic sentential logics have interesting counterparts in the $\pi$-institution framework. The most significant among those are an analog of the characterization of protoalgebraicity in terms of the form of the full models of the logic (Theorem 3.4 of [13]) and an analog of the isomorphism between the lattice of all filters that generate full models and all Alg 8 -congruences (Proposition 3.5 of [13]). Finally, in Section 6, an attempt is made to reconcile the model-theoretic operator approach with the more syntactically flavored, translation-based approach that had been the focus of earlier work ([26], [27], [28]). Given a $\pi$-institution $\ell=\langle$ Sign, SEN, $C\rangle$ with $N$ a category of natural transformations on SEN, an algebraiclike counterpart $\mathcal{L}^{N}(\ell)$, called the $N$-Lindenbaum-Tarski counterpart of $\ell$, is associated with $\ell$. There is a mapping (unfortunately not a translation in general) $\left\langle\mathrm{I}_{\text {Sign }}, \beta\right\rangle$ from $\mathcal{L}^{N}(\ell)$ into $\ell$. This mapping, termed the Blok-Pigozzi mapping, always behaves like a semi-interpretation. The Blok-Pigozzi mapping is used in the final result of the section to provide a partial analog of the more challenging direction (i) $\rightarrow$ (v) of Theorem 1.1.3 of [8].

Finally, it is mentioned that analogs of the classes of equivalential logics of Czelakowski [6] and of weakly algebraizable logics of Czelakowski and Jansana [10] 
for the $\pi$-institution framework will be introduced and investigated also in work currently in progress by the author. For all unexplained categorical notation, the reader is referred to any of the standard references [1], [5], or [19].

\section{Basics on $N$-Protoalgebraicity}

In this section, the basic concepts and results pertaining to $N$-protoalgebraic $\pi$ institutions that were presented in [24], a precursor to the present work, are briefly reviewed. The reader is advised to consult [24] for more details and for the proofs of the results that will be presented here.

Let Sign be a category and SEN : Sign $\rightarrow$ Set a functor. Recall from [25] that the clone of all natural transformations on SEN is defined to be the locally small category with collection of objects $\left\{\mathrm{SEN}^{\alpha}: \alpha\right.$ an ordinal $\}$ and collection of morphisms $\tau: \mathrm{SEN}^{\alpha} \rightarrow \mathrm{SEN}^{\beta} \beta$-sequences of natural transformations $\tau: \mathrm{SEN}^{\alpha} \rightarrow \mathrm{SEN}$. Composition

$$
\mathrm{SEN}^{\alpha} \stackrel{\left\langle\tau_{i}: i<\beta\right\rangle}{\longrightarrow} \mathrm{SEN}^{\beta} \stackrel{\left\langle\sigma_{j}: j<\gamma\right\rangle}{\longrightarrow} \mathrm{SEN}^{\gamma}
$$

is defined by

$$
\left\langle\sigma_{j}: j<\gamma\right\rangle \circ\left\langle\tau_{i}: i<\beta\right\rangle=\left\langle\sigma_{j}\left(\left\langle\tau_{i}: i<\beta\right\rangle\right): j<\gamma\right\rangle .
$$

A subcategory of this category containing all objects of the form $\mathrm{SEN}^{k}$ for $k<\omega$ and all projection morphisms $p^{k, i}: \mathrm{SEN}^{k} \rightarrow \mathrm{SEN}, i<k, k<\omega$, with $p_{\Sigma}^{k, i}: \operatorname{SEN}(\Sigma)^{k} \rightarrow \operatorname{SEN}(\Sigma)$ given by

$$
p_{\Sigma}^{k, i}(\vec{\varphi})=\varphi_{i}, \quad \text { for all } \vec{\varphi} \in \operatorname{SEN}(\Sigma)^{k},
$$

and such that, for every family $\left\{\tau_{i}: \mathrm{SEN}^{k} \rightarrow \mathrm{SEN}: i<l\right\}$ of natural transformations in $N$, the sequence $\left\langle\tau_{i}: i<l\right\rangle: \mathrm{SEN}^{k} \rightarrow \mathrm{SEN}^{l}$ is also in $N$ is referred to as a category of natural transformations on SEN.

Let Sign be a category, SEN : Sign $\rightarrow$ Set be a functor, and $N$ be a category of natural transformations on SEN. Given $\Sigma \in|\mathbf{S i g n}|$, an equivalence relation $\theta_{\Sigma}$ on $\operatorname{SEN}(\Sigma)$ is said to be an $N$-congruence if, for all $\sigma: \operatorname{SEN}^{k} \rightarrow \operatorname{SEN}$ in $N$ and all $\vec{\varphi}, \vec{\psi} \in \operatorname{SEN}(\Sigma)^{k}$,

$$
\vec{\varphi} \theta_{\Sigma}^{k} \vec{\psi} \quad \text { imply } \quad \sigma_{\Sigma}(\vec{\varphi}) \theta_{\Sigma} \sigma_{\Sigma}(\vec{\psi}) .
$$

A collection $\theta=\left\{\left\langle\Sigma, \theta_{\Sigma}\right\rangle: \Sigma \in|\mathbf{S i g n}|\right\}$ is called an equivalence system of SEN if

1. $\theta_{\Sigma}$ is an equivalence relation on $\operatorname{SEN}(\Sigma)$, for all $\Sigma \in|\operatorname{Sign}|$,

2. $\operatorname{SEN}(f)^{2}\left(\theta_{\Sigma_{1}}\right) \subseteq \theta_{\Sigma_{2}}$, for all $\Sigma_{1}, \Sigma_{2} \in|\operatorname{Sign}|, f \in \operatorname{Sign}\left(\Sigma_{1}, \Sigma_{2}\right)$.

If, in addition, $N$ is a category of natural transformations on SEN and $\theta_{\Sigma}$ is an $N$ congruence, for all $\Sigma \in|\mathbf{S i g n}|$, then $\theta$ is said to be an $N$-congruence system of SEN.

Given a $\pi$-institution $\ell=\langle$ Sign, SEN, $C\rangle$, an equivalence system $\theta$ of SEN is called a logical equivalence system of $\ell$ if, for all $\Sigma \in|\operatorname{Sign}|, \varphi, \psi \in \operatorname{SEN}(\Sigma)$,

$$
\langle\varphi, \psi\rangle \in \theta_{\Sigma} \quad \text { implies } \quad C_{\Sigma}(\varphi)=C_{\Sigma}(\psi) .
$$

An $N$-congruence system of SEN is a logical $N$-congruence system of $\ell$ if it is logical as an equivalence system of $\ell$.

It is proven in [25] that the collection of all logical $N$-congruence systems of a given $\pi$-institution $\ell$ forms a complete lattice under signature-wise inclusion and the largest element of the lattice is termed the Tarski $N$-congruence system of $\ell$ and 
denoted by $\widetilde{\Omega}^{N}(\ell)$. Theorem 4 of [25] fully characterizes the Tarski $N$-congruence system of a $\pi$-institution.

Theorem 2.1 (Theorem 4 of [25]) Let $\ell=\left\langle\operatorname{Sign}, \operatorname{SEN},\left\{C_{\Sigma}\right\}_{\Sigma \in \mid \text { Sign } \mid}\right\rangle$ be a $\pi$ institution, $N$ a category of natural transformations on SEN, and $\Sigma \in|\mathbf{S i g n}|$. Then $\langle\varphi, \psi\rangle \in \widetilde{\Omega}_{\Sigma}^{N}(\ell)$ if and only if, for all $\Sigma^{\prime} \in|\operatorname{Sign}|$, all $f \in \operatorname{Sign}\left(\Sigma, \Sigma^{\prime}\right)$, all natural transformations $\tau: \mathrm{SEN}^{k} \rightarrow \operatorname{SEN}$ in $N$, all $\vec{\chi}=\left\langle\chi_{0}, \ldots, \chi_{k-1}\right\rangle \in \operatorname{SEN}\left(\Sigma^{\prime}\right)^{k}$, and all $i<k$,

$$
\begin{aligned}
& C_{\Sigma^{\prime}}\left(\tau_{\Sigma^{\prime}}\left(\chi_{0}, \ldots, \chi_{i-1}, \operatorname{SEN}(f)(\varphi), \chi_{i+1}, \ldots, \chi_{k-1}\right)\right) \\
& \quad=C_{\Sigma^{\prime}}\left(\tau_{\Sigma^{\prime}}\left(\chi_{0}, \ldots, \chi_{i-1}, \operatorname{SEN}(f)(\psi), \chi_{i+1}, \ldots, \chi_{k-1}\right)\right) .
\end{aligned}
$$

Equation (1) will be abbreviated to

$$
C_{\Sigma^{\prime}}\left(\tau_{\Sigma^{\prime}}(\operatorname{SEN}(f)(\varphi), \vec{\chi})\right)=C_{\Sigma^{\prime}}\left(\tau_{\Sigma^{\prime}}(\operatorname{SEN}(f)(\psi), \vec{\chi})\right),
$$

with implicit the understanding that $\operatorname{SEN}(f)(\varphi)$, on the left-hand side, and $\operatorname{SEN}(f)(\psi)$, on the right-hand side, may appear in a different than the first, but in the same, place in both sides of the equation.

Let $\ell=\langle$ Sign, SEN, $C\rangle$ be a $\pi$-institution and $N$ a category of natural transformations on SEN. Recall from [21] that a collection $T=\left\{T_{\Sigma}\right\}_{\Sigma \in \mid \text { Sign| }}$ of subsets $T_{\Sigma} \subseteq \operatorname{SEN}(\Sigma), \Sigma \in|\mathbf{S i g n}|$, is called an axiom system of $\ell$ if

$$
\operatorname{SEN}(f)\left(T_{\Sigma_{1}}\right) \subseteq T_{\Sigma_{2}}, \quad \text { for all } \quad \Sigma_{1}, \Sigma_{2} \in|\operatorname{Sign}|, f \in \operatorname{Sign}\left(\Sigma_{1}, \Sigma_{2}\right) .
$$

An axiom system is called a theory system [23] if, in addition, $T_{\Sigma}$ is a $\Sigma$-theory, for every $\Sigma \in \mid$ Sign $\mid$. A collection $T=\left\{T_{\Sigma}\right\}_{\Sigma \in \mid \text { Sign } \mid}$ of subsets $T_{\Sigma} \subseteq \operatorname{SEN}(\Sigma)$ that do not necessarily satisfy Equation (3) will be called an axiom family (a theory family, if $T_{\Sigma}$ is a $\Sigma$-theory, for every $\left.\Sigma \in|\operatorname{Sign}|\right)$. The collection $\operatorname{ThFam}(\ell)$ of theory families of a $\pi$-institution $\ell$ ordered by signature-wise inclusion forms a complete lattice $\operatorname{ThFam}(\ell)=\langle\operatorname{ThFam}(\ell), \leq\rangle$ and the same holds for the collection $\operatorname{ThSys}(\ell)$ of all theory systems under signature-wise inclusion. Moreover, as was shown in Proposition 2.2 of [24], $\operatorname{ThSys}(\ell)=\langle\operatorname{ThSys}(\ell), \leq\rangle$ forms a complete sublattice of $\operatorname{ThFam}(\ell)$.

An $N$-congruence system $\theta$ on SEN is said to be compatible with the axiom family $T$, if, for all $\Sigma \in|\operatorname{Sign}|, \theta_{\Sigma}$ is compatible with $T_{\Sigma}$ in the usual sense; that is, if, for all $\Sigma \in|\operatorname{Sign}|, \varphi, \psi \in \operatorname{SEN}(\Sigma)$,

$$
\langle\varphi, \psi\rangle \in \theta_{\Sigma} \quad \text { and } \quad \varphi \in T_{\Sigma} \quad \text { imply } \quad \psi \in T_{\Sigma} .
$$

In Proposition 2.3 of [24], it was shown that given a $\pi$-institution $\ell=\langle$ Sign, SEN, $\left.\left\{C_{\Sigma}\right\}_{\Sigma \in \mid \text { Sign } \mid}\right\rangle$ with $N$ a category of natural transformations on SEN and an axiom family $T=\left\{T_{\Sigma}\right\}_{\Sigma \in|\operatorname{Sign}|}$ of $\ell$, the collection $\operatorname{Con}^{N}(T)$ of all $N$-congruence systems of $\ell$ that are compatible with $T$ forms a complete lattice $\operatorname{Con}^{N}(T)$ under signature-wise inclusion. The largest $N$-congruence system on SEN that is compatible with an axiom family $T$ is called the Leibniz $N$-congruence system of $T$ and is denoted by $\Omega^{N}(T)$. Proposition 2.4 of [24] gives a characterization of the Leibniz $N$-congruence system of $T$, analogous to that of Theorem 2.1 for the Tarski $N$ congruence system. The abbreviation convention of Equation (2) is in effect in the condition of Proposition 2.2.

Proposition 2.2 (Proposition 2.4 of [24]) Suppose $\ell=\langle$ Sign, SEN, $C\rangle$ is $a \pi$ institution, $N$ a category of natural transformations on SEN, and $T=\left\{T_{\Sigma}\right\}_{\Sigma \in \mid \text { Sign } \mid}$ 
an axiom family of $\ell$. Then, for all $\Sigma \in|\operatorname{Sign}|, \varphi, \psi \in \operatorname{SEN}(\Sigma),\langle\varphi, \psi\rangle \in \Omega_{\Sigma}^{N}(T)$ if and only if, for all $\Sigma^{\prime} \in|\operatorname{Sign}|, f \in \operatorname{Sign}\left(\Sigma, \Sigma^{\prime}\right), \sigma: \operatorname{SEN}^{k} \rightarrow \operatorname{SEN}$ in $N$, and $\vec{\chi} \in \operatorname{SEN}\left(\Sigma^{\prime}\right)^{k-1}$,

$$
\sigma_{\Sigma^{\prime}}(\operatorname{SEN}(f)(\varphi), \vec{\chi}) \in T_{\Sigma^{\prime}} \quad \text { iff } \quad \sigma_{\Sigma^{\prime}}(\operatorname{SEN}(f)(\psi), \vec{\chi}) \in T_{\Sigma^{\prime}}
$$

A $\pi$-institution $\ell=\langle$ Sign, $\operatorname{SEN}, C\rangle$, with $N$ a category of natural transformations on SEN, was called in [24] $N$-protoalgebraic if, for every theory family $T=\left\{T_{\Sigma}\right\}_{\Sigma \in|\operatorname{Sign}|}$ of $\ell$, every $\Sigma \in|\operatorname{Sign}|$, and all $\varphi, \psi \in \operatorname{SEN}(\Sigma)$,

$$
\langle\varphi, \psi\rangle \in \Omega_{\Sigma}^{N}(T) \text { implies } C_{\Sigma}\left(T_{\Sigma} \cup\{\varphi\}\right)=C_{\Sigma}\left(T_{\Sigma} \cup\{\psi\}\right) .
$$

It was shown in Lemma 3.8 of [24] that the Leibniz operator $\Omega^{N}$, perceived as an operator from the collection of all theory families to the collection of all $N$-congruence systems, is monotonic if and only if $\ell$ is $N$-protoalgebraic. This characterization of $N$-protoalgebraicity forms an analog of the well-known characterization of protoalgebraic logics by Blok and Pigozzi [3]. Moreover, this condition is equivalent to the condition that, for every collection $\left\{T^{i}: i \in I\right\}$ of theory families of $\ell$, $\Omega^{N}\left(\bigcap_{i \in I} T^{i}\right)=\bigcap_{i \in I} \Omega^{N}\left(T^{i}\right)$. (See also [3] and [24] for proofs in the sentential logic and in the $\pi$-institution framework, respectively.)

Some of the basic properties of $N$-protoalgebraic $\pi$-institutions that were proven in [24] will be used in establishing some new properties in the present work. The reader will be referred to the relevant results in [24] whenever necessary.

\section{Implication Systems}

The concept of an $N$-implication system for a $\pi$-institution $\ell$ is now introduced as an analog of the corresponding concept from the theory of protoalgebraic deductive systems; see, for example, [8].

Let $\ell=\langle$ Sign, SEN, $C\rangle$ be a $\pi$-institution and $N$ a category of natural transformations on SEN. A collection $E=\left\{\epsilon^{i}: i \in I\right\}$ of natural transformations $\epsilon^{i}: \mathrm{SEN}^{2} \rightarrow$ SEN in $N$ is said to be an $N$-implication system for $\ell$ if, for every $\Sigma \in|\operatorname{Sign}|$ and all $\varphi, \psi \in \operatorname{SEN}(\Sigma)$,

$$
\begin{aligned}
& \text { 1. } \epsilon_{\Sigma}^{i}(\varphi, \varphi) \in C_{\Sigma}(\varnothing) \text {, for all } i \in I, \quad \text { (E-Reflexivity) } \\
& \text { 2. } \psi \in C_{\Sigma}\left(\left\{\epsilon_{\Sigma}^{i}(\varphi, \psi): i \in I\right\} \cup\{\varphi\}\right) \text {. (E-modus ponens) }
\end{aligned}
$$

Usually these two properties will be abbreviated, respectively, as

$$
E_{\Sigma}(\varphi, \varphi) \subseteq C_{\Sigma}(\varnothing), \quad \psi \in C_{\Sigma}\left(E_{\Sigma}(\varphi, \psi), \varphi\right) .
$$

There exists an interesting relationship between $N$-implication systems for a $\pi$ institution $\ell$ and those subsets (if such subsets exist) of the collection of all natural transformations $\delta: \mathrm{SEN}^{2} \rightarrow$ SEN in $N$ that define the Leibniz $N$-congruence systems of theory families of $\ell$.

Given a $\pi$-institution $\ell=\langle$ Sign, SEN, $C\rangle$ and $N$ a category of natural transformations on SEN, we say that the Leibniz $N$-congruence systems of theory families of $\ell$ are $N$-definable if there exists a subset $D$ of the collection of all natural transformations $\sigma: \mathrm{SEN}^{2} \rightarrow \mathrm{SEN}$ in $N$ such that, for all theory families $T$ of $\ell$ and all $\Sigma \in|\operatorname{Sign}|, \varphi, \psi \subseteq \operatorname{SEN}(\Sigma)$,

$$
\langle\varphi, \psi\rangle \in \Omega_{\Sigma}^{N}(T) \quad \text { iff } \quad \delta_{\Sigma}(\varphi, \psi) \in T_{\Sigma}, \text { for all } \delta \in D .
$$

The condition on the right-hand side of the equivalence above will be abbreviated as $D_{\Sigma}(\varphi, \psi) \subseteq T_{\Sigma}$; that is, Condition (6) becomes $\langle\varphi, \psi\rangle \in \Omega_{\Sigma}^{N}(T)$ if and only if 
$D_{\Sigma}(\varphi, \psi) \subseteq T_{\Sigma}$. If this is the case, $D$ is said to define the Leibniz $N$-congruence systems of the theory families of $\ell$.

Proposition 3.1 Let $\ell=\langle$ Sign, SEN, $C\rangle$ be a $\pi$-institution and $N$ a category of natural transformations on SEN. If $D$ defines the Leibniz $N$-congruence systems of theory families of $\ell$, then $D$ is an $N$-implication system for $\ell$.

Proof Suppose that $D$ defines the Leibniz $N$-congruence systems of theory families of $\ell$. Clearly, for all $\Sigma \in|\operatorname{Sign}|, \varphi \in \operatorname{SEN}(\Sigma)$, we have $\langle\varphi, \varphi\rangle \in \Omega_{\Sigma}^{N}(\mathrm{Thm})$, where by Thm is denoted the theorem system Thm $=\left\{\operatorname{Thm}_{\Sigma}\right\}_{\Sigma \in \mid \text { Sign } \mid}=\left\{C_{\Sigma}(\varnothing)\right\}_{\Sigma \in \mid \text { Sign } \mid}$ of $\ell$. Therefore, for all $\delta \in D, \delta_{\Sigma}(\varphi, \varphi) \in \operatorname{Thm}_{\Sigma}=C_{\Sigma}(\varnothing)$; that is, $D$ satisfies reflexivity.

Now suppose that $\Sigma \in|\operatorname{Sign}|, \varphi, \psi \in \operatorname{SEN}(\Sigma)$. To prove the $N$-modus ponens with respect to $D$, it suffices to show that, for every theory family $T$ of $\ell$, if $D_{\Sigma}(\varphi, \psi) \cup\{\varphi\} \subseteq T_{\Sigma}$, then $\psi \in T_{\Sigma}$. To this end, suppose that $D_{\Sigma}(\varphi, \psi) \subseteq T_{\Sigma}$ and $\varphi \in T_{\Sigma}$. Then $\langle\varphi, \psi\rangle \in \Omega_{\Sigma}^{N}(T)$ and $\varphi \in T_{\Sigma}$, whence, by the compatibility property of $\Omega^{N}(T)$, we get $\psi \in T_{\Sigma}$.

It turns out, similarly with the case of sentential logics (Lemma 2.9 of [3]), that if a $\pi$-institution $\ell$ has an $N$-implication system, then it is $N$-protoalgebraic.

Proposition 3.2 Let $\ell=\langle$ Sign, SEN, $C\rangle$ be a $\pi$-institution and $N$ a category of natural transformations on SEN. If $\ell$ has an $N$-implication system, then it is $N$ protoalgebraic.

Proof Suppose, first, that $\ell$ has an $N$-implication system $E, T$ is a theory family of $\ell$, and $\Sigma \in|\operatorname{Sign}|, \varphi, \psi \in \operatorname{SEN}(\Sigma)$ such that $\langle\varphi, \psi\rangle \in \Omega_{\Sigma}^{N}(T)$. Then since $\Omega^{N}(T)$ is an $N$-congruence system and $E$ consists of natural transformations in $N$, we have that $\left\langle\epsilon_{\Sigma}^{i}(\varphi, \psi), \epsilon_{\Sigma}^{i}(\varphi, \varphi)\right\rangle \in \Omega_{\Sigma}^{N}(T)$, for all $i \in I$. Therefore, since $E_{\Sigma}(\varphi, \varphi) \subseteq C_{\Sigma}(\varnothing) \subseteq T_{\Sigma}$, we get, by the compatibility property of $\Omega^{N}(T)$, $\epsilon_{\Sigma}^{i}(\varphi, \psi) \in T_{\Sigma} \subseteq C_{\Sigma}\left(T_{\Sigma} \cup\{\varphi\}\right)$, for all $i \in I$. Hence, since also $\varphi \in C_{\Sigma}\left(T_{\Sigma} \cup\{\varphi\}\right)$, we get, by $E$-modus ponens,

$$
\psi \in C_{\Sigma}\left(E_{\Sigma}(\varphi, \psi), \varphi\right) \subseteq C_{\Sigma}\left(T_{\Sigma} \cup\{\varphi\}\right) .
$$

By symmetry, $\varphi \in C_{\Sigma}\left(T_{\Sigma} \cup\{\psi\}\right)$ and, therefore, $C_{\Sigma}\left(T_{\Sigma} \cup\{\varphi\}\right)=C_{\Sigma}\left(T_{\Sigma} \cup\{\psi\}\right)$ and $\ell$ is $N$-protoalgebraic.

Finally, combining Proposition 3.2 with Proposition 3.1, we get the following corollary.

Corollary 3.3 Let $\ell=\langle$ Sign, SEN, $C\rangle$ be a $\pi$-institution and $N$ a category of natural transformations on SEN. If the Leibniz $N$-congruence systems of the theory families of $\ell$ are $N$-definable, then $\ell$ is $N$-protoalgebraic.

On some thoughts of why the converse of Proposition 3.2 is not likely to hold for an arbitrary $\pi$-institution, see the discussion concerning parameterized equivalence systems that opens the following section.

Finally, we close the section by discussing a result of [9] characterizing protoalgebraicity of deductive systems in terms of the existence of a parameterized local deduction-detachment theorem. This result can be partially carried over to the $\pi$ institution framework. As was the case with implication systems, the easy direction is carried over in the present context unaltered. Problems remain, for similar reasons 
as with implication systems, if one attempts to move toward a proof of a version of the converse implication. For a more detailed exposition between the connections of protoalgebraicity with the parameterized local deduction-detachment theorem in the case of sentential logics, see Chapter 2 of [8].

Let $\ell=\langle$ Sign, SEN, $C\rangle$ be a $\pi$-institution with $N$ a category of natural transformations on SEN. By analogy with the case of deductive systems, $\ell$ will be said to have the $N$-parameterized local deduction-detachment theorem ( $N$-PLDDT) if there exists a family of sets of natural transformations $\mathcal{E}=\left\{E^{i}: i \in I\right\}$, where $E^{i}=\left\{\epsilon^{i j}: j \in J_{i}\right\}$, with $\epsilon^{i j}: \operatorname{SEN}^{2+k_{i j}} \rightarrow$ SEN in $N$ such that, for all $\Sigma \in|\operatorname{Sign}|, \Phi \cup\{\varphi, \psi\} \subseteq \operatorname{SEN}(\Sigma)$,

$$
\psi \in C_{\Sigma}(\Phi \cup\{\varphi\}) \text { iff }
$$

$$
(\exists i \in I)\left(\exists \vec{\chi} \in \operatorname{SEN}(\Sigma)^{\omega}\right)\left(\forall j \in J_{i}\right) \epsilon_{\Sigma}^{i j}\left(\varphi, \psi, \chi_{0}, \ldots, \chi_{k_{i j}-1}\right) \in C_{\Sigma}(\Phi) .
$$

The notation $\vec{\chi}_{i j}$ will be used in place of $\left\langle\chi_{0}, \ldots, \chi_{k_{i j}-1}\right\rangle$. Similarly with the case of deductive systems, if a $\pi$-institution $\ell$ has an $N$-PLDDT, then it is $N$-protoalgebraic.

Proposition 3.4 Let $\ell=\langle$ Sign, SEN, $C\rangle$ be a $\pi$-institution with $N$ a category of natural transformations on SEN. If $\ell$ has the $N$-PLDDT, then it is $N$-protoalgebraic.

Proof Suppose that $\ell$ has the $N$-PLDDT with respect to the family $\mathcal{E}$. Let $T$ be a theory family of $\ell, \Sigma \in|\operatorname{Sign}|$, and $\varphi, \psi \in \operatorname{SEN}(\Sigma)$ such that $\langle\varphi, \psi\rangle \in \Omega_{\Sigma}^{N}(T)$. Since $\varphi \in C_{\Sigma}(\varphi)$, there exist $i \in I, \vec{\chi} \in \operatorname{SEN}(\Sigma)^{\omega}$ such that $\epsilon_{\Sigma}^{i j}\left(\varphi, \varphi, \vec{\chi}_{i j}\right)$ $\in C_{\Sigma}(\varnothing) \subseteq T_{\Sigma}$, for all $j \in J_{i}$. But $\Omega^{N}(T)$ is an $N$-congruence system, whence, since $\langle\varphi, \psi\rangle \in \Omega_{\Sigma}^{N}(T)$, we get that

$$
\left\langle\epsilon_{\Sigma}^{i j}\left(\varphi, \psi, \vec{\chi}_{i j}\right), \epsilon_{\Sigma}^{i j}\left(\varphi, \varphi, \vec{\chi}_{i j}\right)\right\rangle \in \Omega_{\Sigma}^{N}(T) \text {, for all } j \in J_{i} .
$$

Hence, by the compatibility property of $\Omega^{N}(T)$, we get that $\epsilon_{\Sigma}^{i j}\left(\varphi, \psi, \vec{\chi}_{i j}\right) \in T_{\Sigma}$, for all $j \in J_{i}$. Thus since $\mathcal{E}$ is an $N$-PLDDT family for $\ell$, we conclude that $\psi \in C_{\Sigma}\left(T_{\Sigma} \cup\{\varphi\}\right)$. By symmetry, we get that $\varphi \in C_{\Sigma}\left(T_{\Sigma} \cup\{\psi\}\right)$ and, therefore, $\ell$ is $N$-protoalgebraic.

\section{Parameterized Equivalence Systems}

In Section 1.2 of [8], protoalgebraicity of deductive systems is characterized in terms of the existence of a parameterized system of equivalence sentences. A similar result for $\pi$-institutions will be explored here. Since the context of $\pi$-institutions is very general, a weaker result than Theorem 1.2.7 of [8] is only possible in the present framework. For the sake of comparing the two underlying structures, the reader is reminded that, if a deductive system is cast in the form of a $\pi$-institution, then every sentence of the $\pi$-institution gives rise to a unique natural transformation from sentences to sentences (the term operation defined by the corresponding term in universal algebraic terminology). Similarly, composition of natural transformations is reflected in substituting corresponding terms for variables in corresponding terms. This interaction between sentences and natural transformations is not necessarily present in an arbitrary $\pi$-institution. This is one of the main reasons why additional assumptions have to be made in order to get results comparable in power to the ones pertaining to deductive systems.

Let $\ell=\langle$ Sign, SEN, $C\rangle$ be a $\pi$-institution and $N$ a category of natural transformations on SEN. Suppose that $E$ is a set of natural transformations of the form 
$\epsilon: \mathrm{SEN}^{k+2} \rightarrow \mathrm{SEN}$ in $N$, for some $k \in \omega, k$ possibly varying from transformation to transformation. The following notation, borrowed from [8], will prove convenient.

$$
E_{\Sigma}(\varphi, \psi, \vec{\chi})=\left\{\epsilon_{\Sigma}\left(\varphi, \psi, \chi_{0}, \ldots, \chi_{k-1}\right): \epsilon \in E \text { with } \epsilon: \operatorname{SEN}^{k+2} \rightarrow \operatorname{SEN}\right\},
$$

for all $\Sigma \in|\operatorname{Sign}|, \varphi, \psi \in \operatorname{SEN}(\Sigma)$, and $\vec{\chi} \in \operatorname{SEN}(\Sigma)^{\omega}$. Also, following [8], Section 1.2 , denote, for all $\Sigma \in|\operatorname{Sign}|, \varphi, \psi \in \operatorname{SEN}(\Sigma)$,

$$
E_{\Sigma}(\langle\varphi, \psi\rangle)=\bigcup_{\vec{\chi} \in \operatorname{SEN}(\Sigma)^{\omega}} E_{\Sigma}(\varphi, \psi, \vec{\chi}) .
$$

Finally, for all theory families $T$ of $\ell$, define the family of binary relations $E(T)=\left\{E_{\Sigma}(T)\right\}_{\Sigma \in \mid \text { Sign } \mid}$ by letting, for all $\Sigma \in|\operatorname{Sign}|, \varphi, \psi \in \operatorname{SEN}(\Sigma)$,

$$
\begin{array}{r}
\langle\varphi, \psi\rangle \in E_{\Sigma}(T) \quad \text { iff } \quad E_{\Sigma^{\prime}}(\langle\operatorname{SEN}(f)(\varphi), \operatorname{SEN}(f)(\psi)\rangle) \subseteq T_{\Sigma^{\prime}}, \\
\text { for all } \Sigma^{\prime} \in|\operatorname{Sign}|, f \in \operatorname{Sign}\left(\Sigma, \Sigma^{\prime}\right) .
\end{array}
$$

It is easy to see that, for all $\Sigma_{1}, \Sigma_{2} \in|\operatorname{Sign}|, f \in \operatorname{Sign}\left(\Sigma_{1}, \Sigma_{2}\right)$,

$$
\langle\varphi, \psi\rangle \in E_{\Sigma_{1}}(T) \quad \text { implies }\langle\operatorname{SEN}(f)(\varphi), \operatorname{SEN}(f)(\psi)\rangle \in E_{\Sigma_{2}}(T) ;
$$

that is, $E(T)$ is a relation system (in analogy with equivalence and theory systems) on SEN.

If $E(T)$ is a reflexive relation system, that is, $E_{\Sigma}(T)$ is a reflexive binary relation on $\operatorname{SEN}(\Sigma)$, for all $\Sigma \in|\operatorname{Sign}|$, then it contains the Leibniz congruence system $\Omega(T)$. This is the analog of Proposition 1.2.1 of [8] (see also Theorem 1.6 of [4]).

Proposition 4.1 Let $\ell=\langle$ Sign, $\mathrm{SEN}, C\rangle$ be a $\pi$-institution, $N$ a category of natural transformations on SEN, and $T$ a theory family of $\ell$. If $E(T)$ is a reflexive relation system on $\mathrm{SEN}$, then $\Omega^{N}(T) \leq E(T)$.

Proof Let $\Sigma \in|\mathbf{S i g n}|, \varphi, \psi \in \operatorname{SEN}(\Sigma)$ such that $\langle\varphi, \psi\rangle \in \Omega_{\Sigma}^{N}(T)$. Then, for all $\Sigma^{\prime} \in|\operatorname{Sign}|, f \in \operatorname{Sign}\left(\Sigma, \Sigma^{\prime}\right), \epsilon: \operatorname{SEN}^{k+2} \rightarrow \operatorname{SEN}$ in $E$, and all $\vec{\chi} \in \operatorname{SEN}\left(\Sigma^{\prime}\right)^{k}$, we have that

$$
\left\langle\epsilon_{\Sigma^{\prime}}(\operatorname{SEN}(f)(\varphi), \operatorname{SEN}(f)(\psi), \vec{\chi}), \epsilon_{\Sigma^{\prime}}(\operatorname{SEN}(f)(\varphi), \operatorname{SEN}(f)(\varphi), \vec{\chi})\right\rangle \in \Omega_{\Sigma^{\prime}}^{N}(T) .
$$

But, by the reflexivity of $E(T), \epsilon_{\Sigma^{\prime}}(\operatorname{SEN}(f)(\varphi), \operatorname{SEN}(f)(\varphi), \vec{\chi}) \in T_{\Sigma^{\prime}}$, whence, by the compatibility of $\Omega^{N}(T)$, we obtain $\epsilon_{\Sigma^{\prime}}(\operatorname{SEN}(f)(\varphi), \operatorname{SEN}(f)(\psi), \vec{\chi}) \in T_{\Sigma^{\prime}}$. Thus, $\langle\varphi, \psi\rangle \in E_{\Sigma}(T)$ and, therefore, $\Omega^{N}(T) \leq E(T)$.

Proposition 4.1 implies that, if $E(T)$ is an $N$-congruence system of $\ell$ compatible with the theory family $T$, then it has to coincide with $\Omega^{N}(T)$.

Corollary 4.2 Let $\ell=\langle$ Sign, SEN, $C\rangle$ be a $\pi$-institution, $N$ a category of natural transformations on SEN, and $T$ a theory family of $\ell$. If $E(T)$ is an $N$-congruence system on SEN that is compatible with $T$, then $E(T)=\Omega^{N}(T)$.

Given a $\pi$-institution $\ell=\langle$ Sign, SEN,$C\rangle$ and $N$ a category of natural transformations on SEN, a subset $E$ of $N$, as above, will be said to be an $N$-parameterized equivalence system for $\ell$ if, for all $\Sigma \in|\mathbf{S i g n}|, \sigma: \operatorname{SEN}^{n} \rightarrow \operatorname{SEN}$ in $N$, and all $\vec{\varphi}, \vec{\psi} \in \operatorname{SEN}(\Sigma)^{n}$,

(R) $E_{\Sigma}(\langle\varphi, \varphi\rangle) \subseteq C_{\Sigma}(\varnothing)$;

(MP) for every theory family $T$ of $\ell$,

(a) $\varphi \in T_{\Sigma}$ and 
(b) $E_{\Sigma^{\prime}}(\langle\operatorname{SEN}(f)(\varphi), \operatorname{SEN}(f)(\psi)\rangle) \subseteq T_{\Sigma^{\prime}}$, for all $\Sigma^{\prime} \in|\operatorname{Sign}|$ and all $f \in \operatorname{Sign}\left(\Sigma, \Sigma^{\prime}\right)$,

imply $\psi \in T_{\Sigma}$;

(RP) for every theory family $T$ of $\ell, E_{\Sigma^{\prime}}\left(\left\langle\operatorname{SEN}(f)\left(\varphi_{i}\right), \operatorname{SEN}(f)\left(\psi_{i}\right)\right\rangle\right) \subseteq T_{\Sigma^{\prime}}$, for all $\Sigma^{\prime} \in|\mathbf{S i g n}|$ and all $f \in \operatorname{Sign}\left(\Sigma, \Sigma^{\prime}\right), i<n$, imply

$$
E_{\Sigma^{\prime}}\left(\left\langle\operatorname{SEN}(f)\left(\sigma_{\Sigma}(\vec{\varphi})\right), \operatorname{SEN}(f)\left(\sigma_{\Sigma}(\vec{\psi})\right)\right\rangle\right) \subseteq T_{\Sigma^{\prime}},
$$

for all $\Sigma^{\prime} \in|\operatorname{Sign}|$ and all $f \in \operatorname{Sign}\left(\Sigma, \Sigma^{\prime}\right)$.

(R) stands for reflexivity, (MP) for modus ponens, and (RP) for replacement. Note the difference between these conditions and the corresponding conditions for the parameterized equivalence systems of [8]. The added complexity here is due to the effort to make the collection of relations $E(T)$ structural, that is, a relation system.

The following lemma asserts that an $N$-parameterized equivalence system $E$ of a $\pi$-institution $\ell$ gives rise to a reflexive and $N$-invariant relation system $E(T)$ that is compatible with $T$, for every theory family $T$ of $\ell$. This parallels Claim 1 of Theorem 1.2.4 of [8].

Lemma 4.3 Let $\ell=\langle$ Sign, SEN, $C\rangle$ be a $\pi$-institution and $N$ a category of natural transformations on SEN. If $E$ is an $N$-parameterized equivalence system for $l$ and $T$ a theory family of $\ell$, then

1. $E(T)$ is reflexive,

2. $E(T)$ is compatible with $T$, and

3. for all $\Sigma \in|\operatorname{Sign}|, \sigma: \operatorname{SEN}^{n} \rightarrow \operatorname{SEN}$ in $N, \vec{\varphi}, \vec{\psi} \in \operatorname{SEN}(\Sigma)^{n}$,

$$
\left\langle\varphi_{i}, \psi_{i}\right\rangle \in E_{\Sigma}(T), i<n, \quad \text { imply }\left\langle\sigma_{\Sigma}(\vec{\varphi}), \sigma_{\Sigma}(\vec{\psi})\right\rangle \in E_{\Sigma}(T) .
$$

\section{Proof}

1 Since $E$ is an $N$-parameterized equivalence system of $\ell$, we get that, for all $\Sigma^{\prime} \in|\operatorname{Sign}|, f \in \operatorname{Sign}\left(\Sigma, \Sigma^{\prime}\right), E_{\Sigma^{\prime}}(\langle\operatorname{SEN}(f)(\varphi), \operatorname{SEN}(f)(\varphi)\rangle) \subseteq C_{\Sigma^{\prime}}(\varnothing) \subseteq T_{\Sigma^{\prime}}$. Therefore $\langle\varphi, \varphi\rangle \in E_{\Sigma}(T)$ and $E(T)$ is reflexive.

2 Suppose that $\langle\varphi, \psi\rangle \in E_{\Sigma}(T)$ and $\varphi \in T_{\Sigma}$. Then, for all $\Sigma^{\prime} \in \mid$ Sign $\mid$, $f \in \operatorname{Sign}\left(\Sigma, \Sigma^{\prime}\right), E_{\Sigma^{\prime}}(\langle\operatorname{SEN}(f)(\varphi), \operatorname{SEN}(f)(\psi)\rangle) \subseteq T_{\Sigma^{\prime}}$, and $\varphi \in T_{\Sigma}$, whence, since $E$ is an $N$-parameterized equivalence system, $\psi \in T_{\Sigma}$ as well; that is, $E(T)$ is compatible with $T$.

3 Finally, suppose that $\Sigma \in|\operatorname{Sign}|, \sigma: \operatorname{SEN}^{n} \rightarrow \operatorname{SEN}$ in $N$, and $\vec{\varphi}, \vec{\psi} \in \operatorname{SEN}(\Sigma)^{n}$ such that $\left\langle\varphi_{i}, \psi_{i}\right\rangle \in E_{\Sigma}(T)$, for all $i<n$. Then, for all $\Sigma^{\prime} \in|\operatorname{Sign}|, f \in \operatorname{Sign}\left(\Sigma, \Sigma^{\prime}\right)$, $E_{\Sigma^{\prime}}\left(\left\langle\operatorname{SEN}(f)\left(\varphi_{i}\right), \operatorname{SEN}(f)\left(\psi_{i}\right)\right\rangle\right) \subseteq T_{\Sigma^{\prime}}$, whence, since $E$ is an $N$-parameterized equivalence system, we get that $E_{\Sigma^{\prime}}\left(\left\langle\operatorname{SEN}(f)\left(\sigma_{\Sigma}(\vec{\varphi})\right), \operatorname{SEN}(f)\left(\sigma_{\Sigma}(\vec{\psi})\right)\right\rangle\right) \subseteq T_{\Sigma^{\prime}}$, which yields that $\left\langle\sigma_{\Sigma}(\vec{\varphi}), \sigma_{\Sigma}(\vec{\psi})\right\rangle \in E_{\Sigma}(T)$.

Next it is shown that the relation $E(T)$, associated with an $N$-parameterized equivalence system $E$ of a $\pi$-institution $\ell$ and a theory family $T$ of $\ell$, satisfies a property satisfied by all $N$-congruence systems of $\ell$. Roughly speaking, this property may be described as a generalized replacement property that allows the substitution of related sentences into the same context modulo any signature morphism. In the statement of Lemma 4.4, the notational convention of Equation (2) is in effect.

Lemma 4.4 Let $\ell=\langle$ Sign, SEN, $C\rangle$ be a $\pi$-institution with $N$ a category of natural transformations on SEN. If $E$ is an $N$-parameterized equivalence system 
for $\ell$ and $T$ a theory family of $\ell$, then, for all $\Sigma \in|\operatorname{Sign}|, \varphi, \psi \in \operatorname{SEN}(\Sigma)$, $\langle\varphi, \psi\rangle \in E_{\Sigma}(T)$ implies that, for all $\Sigma^{\prime} \in|\operatorname{Sign}|, f \in \operatorname{Sign}\left(\Sigma, \Sigma^{\prime}\right)$, and all $\sigma: \operatorname{SEN}^{n} \rightarrow \operatorname{SEN}$ in $N, \vec{\chi} \in \operatorname{SEN}\left(\Sigma^{\prime}\right)^{n-1}$,

$$
\left\langle\sigma_{\Sigma^{\prime}}(\operatorname{SEN}(f)(\varphi), \vec{\chi}), \sigma_{\Sigma^{\prime}}(\operatorname{SEN}(f)(\psi), \vec{\chi})\right\rangle \in E_{\Sigma^{\prime}}(T) .
$$

Proof Suppose that $\Sigma \in|\mathbf{S i g n}|$ and $\varphi, \psi \in \operatorname{SEN}(\Sigma)$ such that $\langle\varphi, \psi\rangle \in E_{\Sigma}(T)$. Then since $E(T)$ is a relation system on SEN (see remark preceding Proposition 4.1), for every $\Sigma^{\prime} \in|\operatorname{Sign}|, f \in \operatorname{Sign}\left(\Sigma, \Sigma^{\prime}\right),\langle\operatorname{SEN}(f)(\varphi), \operatorname{SEN}(f)(\psi)\rangle$ $\in E_{\Sigma^{\prime}}(T)$. Thus since by Lemma 4.3, Part $1, E(T)$ is reflexive, for all $\sigma: \operatorname{SEN}^{n} \rightarrow$ SEN in $N, \vec{\chi} \in \operatorname{SEN}\left(\Sigma^{\prime}\right)^{n-1}$, we obtain, by Lemma 4.3, Part 3, $\left\langle\sigma_{\Sigma^{\prime}}(\operatorname{SEN}(f)(\varphi), \vec{\chi}), \sigma_{\Sigma^{\prime}}(\operatorname{SEN}(f)(\psi), \vec{\chi})\right\rangle \in E_{\Sigma^{\prime}}(T)$.

In the next lemma it is shown that $E(T)$ is a symmetric relation system of $\ell$ for every $N$-parameterized equivalence system $E$ and every theory family $T$ of $\ell$.

Lemma 4.5 Let $\ell=\langle$ Sign, SEN, $C\rangle$ be a $\pi$-institution with $N$ a category of natural transformations on SEN. If $E$ is an $N$-parameterized equivalence system for $\ell$ and $T$ a theory family of $\ell$, then $E(T)$ is symmetric.

Proof Suppose that $\langle\varphi, \psi\rangle \in E_{\Sigma}(T)$. Then, on the one hand, for all $\Sigma^{\prime} \in|\operatorname{Sign}|$, $f \in \operatorname{Sign}\left(\Sigma, \Sigma^{\prime}\right), E_{\Sigma^{\prime}}(\langle\operatorname{SEN}(f)(\varphi), \operatorname{SEN}(f)(\psi)\rangle) \subseteq T_{\Sigma^{\prime}}$, and, on the other, by Lemma 4.4, for every $\epsilon \in E, \vec{\chi} \in \operatorname{SEN}\left(\Sigma^{\prime}\right)^{k}$,

$$
\left\langle\epsilon_{\Sigma^{\prime}}(\operatorname{SEN}(f)(\varphi), \operatorname{SEN}(f)(\varphi), \vec{\chi}), \epsilon_{\Sigma^{\prime}}(\operatorname{SEN}(f)(\psi), \operatorname{SEN}(f)(\varphi), \vec{\chi})\right\rangle \in E_{\Sigma^{\prime}}(T),
$$

whence, since by reflexivity, $\epsilon_{\Sigma^{\prime}}(\operatorname{SEN}(f)(\varphi), \operatorname{SEN}(f)(\varphi), \vec{\chi}) \in T_{\Sigma^{\prime}}$, we obtain, by Lemma 4.3, Part 2, that $\epsilon_{\Sigma^{\prime}}(\operatorname{SEN}(f)(\psi), \operatorname{SEN}(f)(\varphi), \vec{\chi}) \in T_{\Sigma^{\prime}}$. Thus $\langle\psi, \varphi\rangle \in E_{\Sigma}(T)$ and $E(T)$ is symmetric.

The next theorem is a partial analog of Theorem 1.2.4 of [8]. It shows that a collection $E$ of natural transformations in $N$ is an $N$-parameterized equivalence system for a $\pi$-institution $\ell$ if and only if $E(T)=\Omega^{N}(T)$, for every theory family $T$ of $\ell$.

Theorem 4.6 Let $\ell=\langle$ Sign, SEN, $C\rangle$ be a $\pi$-institution with $N$ a category of natural transformations on SEN. $E$ is an $N$-parameterized equivalence system for $\ell$ if and only if, for every theory family $T$ of $\ell, E(T)=\Omega^{N}(T)$.

Proof Suppose that $E$ is an $N$-parameterized equivalence system for $\ell$. Since by Lemma 4.3 $E(T)$ is reflexive, we obtain, by Proposition 4.1, that $\Omega^{N}(T) \leq E(T)$. On the other hand, if $\Sigma \in|\operatorname{Sign}|, \varphi, \psi \in \operatorname{SEN}(\Sigma)$ such that $\langle\varphi, \psi\rangle \in E_{\Sigma}(T)$, then, for every $\Sigma^{\prime} \in|\operatorname{Sign}|, f \in \operatorname{Sign}\left(\Sigma, \Sigma^{\prime}\right), \sigma: \operatorname{SEN}^{n} \rightarrow$ SEN in $N$, and $\vec{\chi} \in \operatorname{SEN}\left(\Sigma^{\prime}\right)^{n-1}$, we get, by Lemma 4.4, taking into account once more the notational convention of Equation (2),

$$
\left\langle\sigma_{\Sigma^{\prime}}(\operatorname{SEN}(f)(\varphi), \vec{\chi}), \sigma_{\Sigma^{\prime}}(\operatorname{SEN}(f)(\psi), \vec{\chi})\right\rangle \in E_{\Sigma^{\prime}}(T) .
$$

Therefore, since $E$ is an $N$-parameterized equivalence system for $\ell$, it is compatible with $T$, by Lemma 4.3, Part 2, and it is symmetric, by Lemma 4.5, whence we obtain that

$$
\sigma_{\Sigma^{\prime}}(\operatorname{SEN}(f)(\varphi), \vec{\chi}) \in T_{\Sigma^{\prime}} \quad \text { iff } \quad \sigma_{\Sigma^{\prime}}(\operatorname{SEN}(f)(\psi), \vec{\chi}) \in T_{\Sigma^{\prime}}
$$

Thus, by Proposition 4 of [24], characterizing the Leibniz $N$-congruence system of $\ell$ associated with a theory family $T$ of $\ell,\langle\varphi, \psi\rangle \in \Omega_{\Sigma}^{N}(T)$. Therefore $E(T) \leq \Omega^{N}(T)$. 
Suppose, conversely, that $E$ is a family of natural transformations in $N$ such that, for every theory family $T$ of $\ell, E(T)=\Omega^{N}(T)$. Then $E(T)$ is reflexive, compatible with $T$, and $N$-invariant, since $\Omega^{N}(T)$ has these properties. Therefore, we have, for all $\Sigma \in|\operatorname{Sign}|, \varphi, \psi \in \operatorname{SEN}(\Sigma), \sigma: \operatorname{SEN}^{n} \rightarrow \operatorname{SEN}$ in $N$, and $\vec{\varphi}, \vec{\psi} \in \operatorname{SEN}(\Sigma)^{n}$,

1. $\langle\varphi, \varphi\rangle \in E_{\Sigma}(\varnothing)$, whence $E_{\Sigma}(\langle\varphi, \varphi\rangle) \subseteq C_{\Sigma}(\varnothing)$ and $E$ satisfies reflexivity.

2. If $\varphi \in T_{\Sigma}$ and, for all $\Sigma^{\prime} \in|\operatorname{Sign}|, f \in \operatorname{Sign}\left(\Sigma, \Sigma^{\prime}\right)$,

$$
E_{\Sigma^{\prime}}(\langle\operatorname{SEN}(f)(\varphi), \operatorname{SEN}(f)(\psi)\rangle) \subseteq T_{\Sigma^{\prime}},
$$

then we have that $\varphi \in T_{\Sigma}$ and $\langle\varphi, \psi\rangle \in E_{\Sigma}(T)=\Omega_{\Sigma}^{N}(T)$, whence $\psi \in T_{\Sigma}$. Thus, modus ponens is also satisfied.

3. Suppose that $E_{\Sigma^{\prime}}\left(\left\langle\operatorname{SEN}(f)\left(\varphi_{i}\right), \operatorname{SEN}(f)\left(\psi_{i}\right)\right\rangle \subseteq T_{\Sigma^{\prime}}\right.$, for all $\Sigma^{\prime} \in|\operatorname{Sign}|$ and all $f \in \operatorname{Sign}\left(\Sigma, \Sigma^{\prime}\right), i<n$. Then we have that $\left\langle\varphi_{i}, \psi_{i}\right\rangle \in E_{\Sigma}(T)$, for all $i<n$, whence, since $E(T)=\Omega^{N}(T)$, we get that

$$
\left\langle\sigma_{\Sigma}(\vec{\varphi}), \sigma_{\Sigma}(\vec{\psi})\right\rangle \in E_{\Sigma}(T) .
$$

This yields that $E_{\Sigma^{\prime}}\left(\left\langle\operatorname{SEN}(f)\left(\sigma_{\Sigma}(\vec{\varphi})\right), \operatorname{SEN}(f)\left(\sigma_{\Sigma}(\vec{\psi})\right)\right\rangle\right) \subseteq T_{\Sigma^{\prime}}$. Thus, replacement also holds.

Since reflexivity, modus ponens, and replacement all hold for $E, E$ is an $N$ parameterized equivalence system for $\ell$.

Finally, in a partial analog of Theorem 1.2.7 of [8], it is shown that a $\pi$-institution $\ell=\langle$ Sign, SEN, $C\rangle$, with $N$ a category of natural transformations on SEN, is $N$ protoalgebraic if it possesses an $N$-parameterized equivalence system. We were unable to prove a version of the converse of Theorem 1.2.7 of [8] in the present framework. The key missing property that allows the proof to be carried out in the deductive system case is the property $p \equiv q\left(\bmod \Omega E_{C}\right)$ (see Lemma 1.1.2 of [8]) and it is impossible to prove an analog of this property for $\pi$-institutions unless there is a link between the sentences of the $\pi$-institution and the natural transformations in $N$. Such a link does indeed exist in the case of deductive systems. On this point, see also the remarks made at the beginning of this section.

Proposition 4.7 Let $\ell=\langle$ Sign, SEN, $C\rangle$ be a $\pi$-institution with $N$ a category of natural transformations on SEN. If l possesses an $N$-parameterized equivalence system, then it is $N$-protoalgebraic.

Proof Suppose that $E$ is an $N$-parameterized equivalence system for $\ell, T$ is a theory family of $\ell$ and $\Sigma \in|\operatorname{Sign}|, \varphi, \psi \in \operatorname{SEN}(\Sigma)$ such that $\langle\varphi, \psi\rangle \in \Omega_{\Sigma}^{N}(T)$. Then since $\Omega^{N}(T)$ is an $N$-congruence system, we have, for all $\Sigma^{\prime} \in|\mathbf{S i g n}|$, $f \in \operatorname{Sign}\left(\Sigma, \Sigma^{\prime}\right)$, and all $\epsilon: \operatorname{SEN}^{k+2} \rightarrow \operatorname{SEN}$ in $E, \vec{\chi} \in \operatorname{SEN}\left(\Sigma^{\prime}\right)^{k}$,

$$
\left\langle\epsilon_{\Sigma^{\prime}}(\operatorname{SEN}(f)(\varphi), \operatorname{SEN}(f)(\psi), \vec{\chi}), \epsilon_{\Sigma^{\prime}}(\operatorname{SEN}(f)(\varphi), \operatorname{SEN}(f)(\varphi), \vec{\chi})\right\rangle \in \Omega_{\Sigma^{\prime}}^{N}(T) .
$$

Therefore, since by reflexivity it holds that

$$
\epsilon_{\Sigma^{\prime}}(\operatorname{SEN}(f)(\varphi), \operatorname{SEN}(f)(\varphi), \vec{\chi}) \in C_{\Sigma^{\prime}}(\varnothing) \subseteq T_{\Sigma^{\prime}},
$$

we get, by the compatibility property of $\Omega^{N}(T)$,

$$
\epsilon_{\Sigma^{\prime}}(\operatorname{SEN}(f)(\varphi), \operatorname{SEN}(f)(\psi), \vec{\chi}) \in T_{\Sigma^{\prime}} .
$$

Hence, by the modus ponens of $E, \psi \in C_{\Sigma}\left(T_{\Sigma} \cup\{\varphi\}\right)$. By symmetry, $\varphi$ $\in C_{\Sigma}\left(T_{\Sigma} \cup\{\psi\}\right)$ and, therefore, $C_{\Sigma}\left(T_{\Sigma} \cup\{\varphi\}\right)=C_{\Sigma}\left(T_{\Sigma} \cup\{\psi\}\right)$ and $\ell$ is $N$ protoalgebraic. 


\section{Semantic Consequences of Protoalgebraicity}

In this section, the direction of our studies changes. Whereas in the preceding sections the "syntactic" aspects of the $\pi$-institution were at the focus of our investigations, the study of the Leibniz $N$-congruences and of the full models started in [24] will be revisited in this section. As a result, quite a few references will be made to results from [24].

By putting together Proposition 5.27 of [24] and the Isomorphism Theorem 13 of [30], it is possible to show that the theorem system of a full model of a protoalgebraic $\pi$-institution via a surjective logical morphism uniquely determines the full model. This is an analog of Lemma 3.3 of [13] for $\pi$-institutions. Recall that by Thm is denoted the theorem system of a $\pi$-institution $\ell$.

Lemma 5.1 Let $\ell=\langle$ Sign, SEN,$C\rangle$ be a $\pi$-institution with $N$ a category of natural transformations on SEN. If $\ell$ is $N$-protoalgebraic and $\ell^{\prime}=\left\langle\mathbf{S i g n}^{\prime}, \mathrm{SEN}^{\prime}, C^{\prime}\right\rangle$, $\ell^{\prime \prime}=\left\langle\mathbf{S i g n}^{\prime}, \mathrm{SEN}^{\prime}, C^{\prime \prime}\right\rangle$ are two full $\left(N, N^{\prime}\right)$-models of $\ell$ via the same surjective $\left(N, N^{\prime}\right)$-logical morphism $\langle F, \alpha\rangle: \mathrm{SEN} \rightarrow{ }^{\text {se }} \mathrm{SEN}^{\prime}$ that have identical theorem systems $\mathrm{Thm}^{\prime}=\mathrm{Thm}^{\prime \prime}$, then $\ell^{\prime}=\ell^{\prime \prime}$.

Proof First note that the surjectivity of $\langle F, \alpha\rangle$ and Proposition 5.27 of [24] imply that both $\ell^{\prime}$ and $\ell^{\prime \prime}$ are $N^{\prime}$-protoalgebraic. Thus, taking Proposition 3.11 of [24] into account and applying the Tarski operator on the full models, we get

$$
\widetilde{\Omega}^{N^{\prime}}\left(C^{\prime}\right)=\Omega^{N^{\prime}}\left(\mathrm{Thm}^{\prime}\right)=\Omega^{N^{\prime}}\left(\mathrm{Thm}^{\prime \prime}\right)=\widetilde{\Omega}^{N^{\prime}}\left(C^{\prime \prime}\right) .
$$

Now since both $\ell^{\prime}$ and $\ell^{\prime \prime}$ are assumed to be full models of $\ell$ via $\langle F, \alpha\rangle$, the Isomorphism Theorem 13 of [30] may be readily applied to give $\ell^{\prime}=\ell^{\prime \prime}$.

Font and Jansana provide in [13], Theorem 3.4, another characterization of protoalgebraicity in terms of the form of the full models of a sentential logic. An analog is given here characterizing $N$-protoalgebraicity which uses the form that is assumed by the full models of the $\pi$-institution via surjective logical morphisms. Roughly speaking, a $\pi$-institution is $N$-protoalgebraic if and only if all its $\left(N, N^{\prime}\right)$-full models via surjective logical morphisms $\langle F, \alpha\rangle$ have closure operators of the form $C^{\prime \mathrm{min}^{T}}$, where $C^{\prime \text { min }}$ is the closure operator of the corresponding $\langle F, \alpha\rangle$-min $\left(N, N^{\prime}\right)$-model and $T$ is one of the theory systems of the $\langle F, \alpha\rangle$-min $\left(N, N^{\prime}\right)$-model. This is the analog of Theorem 3.4 of [13] for $N$-protoalgebraic $\pi$-institutions.

Theorem 5.2 Let $\ell=\langle$ Sign, SEN, $C\rangle$ be a $\pi$-institution with $N$ a category of natural transformations on SEN. $\ell$ is $N$-protoalgebraic if and only if, for every functor $\mathrm{SEN}^{\prime}:$ Sign' $\rightarrow$ Set, every category $N^{\prime}$ of natural transformations $N^{\prime}$ on $\mathrm{SEN}^{\prime}$, and every surjective $\left(N, N^{\prime}\right)$-epimorphic translation $\langle F, \alpha\rangle: \mathrm{SEN} \rightarrow{ }^{\text {se }} \mathrm{SEN}^{\prime}$, all $\langle F, \alpha\rangle$-full $\left(N, N^{\prime}\right)$-models of $\ell$ have the form $\left\langle\mathbf{S i g n}^{\prime}, \mathrm{SEN}^{\prime}, C^{\prime \min ^{\mathrm{T}}}{ }^{\mathrm{T}}\right.$, where $T$ is a theory system of the $\langle F, \alpha\rangle$-min $\left(N, N^{\prime}\right)$-model $\ell^{\prime \text { min }}=\left\langle\mathbf{S i g n}^{\prime}, \mathrm{SEN}^{\prime}, C^{\prime \text { min }}\right\rangle$ of $\ell$ on SEN'.

Proof Suppose, first, that the given condition is true. Let $T^{1}, T^{2}$ be two theory families of $\ell$ such that $T^{1} \leq T^{2}$. Since, by Proposition 5.23 of [24], $\operatorname{Alg}^{N}(\ell)^{*} \subseteq \operatorname{Alg}^{N}(\ell)$, we have that $\Omega^{N}\left(T^{1}\right) \in \operatorname{Con}_{\operatorname{Alg}^{N}(\ell)}^{\left\langle I_{\operatorname{Sign}}, l\right\rangle}$ (SEN). Now, by the Isomorphism Theorem 13 of [30], there exists an $(N, N)$-full model $\ell^{\prime}=\langle$ Sign, SEN, $\left.C^{\prime}\right\rangle$ of $\ell$ on SEN via the identity $(N, N)$-logical morphism $\left\langle\mathrm{I}_{\text {Sign }}, l\right\rangle$ such that 
$\Omega^{N}\left(T^{1}\right)=\widetilde{\Omega}^{N}\left(C^{\prime}\right)$. Since $\ell^{\prime}$ is an $(N, N)$-full model of $\ell$ via the identity, there exists by hypothesis a theory system $T$ of $\ell$ which is the $\left\langle\operatorname{I}_{\operatorname{Sign}}, l\right\rangle$-min $(N, N)$-model of $\ell$ such that $C^{\prime}=C^{T}$. Note that $T^{2} \geq T^{1} \geq T$, since $T^{1} / \Omega^{N}\left(T^{1}\right)$ is a theory fam-

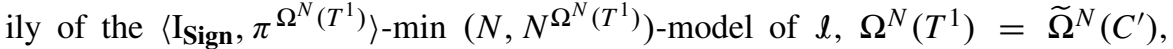
and $\ell^{\prime}$ is full. Now we have $\Omega^{N}\left(T^{1}\right)=\widetilde{\Omega}^{N}\left(C^{\prime}\right) \leq \Omega^{N}\left(T^{2}\right)$. Hence $\Omega^{N}$ is monotonic on the theory families of $\ell$, whence $\ell$ is $N$-protoalgebraic by Lemma 3.8 of [24].

Suppose conversely that $\ell$ is $N$-protoalgebraic and let $\mathrm{SEN}^{\prime}:$ Sign' $^{\prime} \rightarrow$ Set be a functor, $N^{\prime}$ a category of natural transformations on $\mathrm{SEN}^{\prime}$, and $\langle F, \alpha\rangle$ : SEN $\rightarrow{ }^{\text {se }} \mathrm{SEN}^{\prime}$ a surjective $\left(N, N^{\prime}\right)$-epimorphic translation. Moreover, suppose that $\ell^{\prime}=\left\langle\mathbf{S i g n}^{\prime}, \mathrm{SEN}^{\prime}, C^{\prime}\right\rangle$ is an $\langle F, \alpha\rangle$-full $\left(N, N^{\prime}\right)$-model of $\ell$ on $\mathrm{SEN}^{\prime}$ and that $\ell^{\prime \text { min }}=\left\langle\mathbf{S i g n}^{\prime}, \mathrm{SEN}^{\prime}, C^{\prime \text { min }}\right\rangle$ is the $\langle F, \alpha\rangle$-min $\left(N, N^{\prime}\right)$-model of $\ell$ on $\mathrm{SEN}^{\prime}$. Let $\mathrm{Thm}^{\prime}$ be the theorem system of $\ell^{\prime}$. Then since by the definition of a min model $C^{\prime \text { min }} \leq C^{\prime}$, we get that $\mathrm{Thm}^{\prime}$ is a theory system of $\ell^{\prime \text { min }}$ and, obviously, $C^{\prime \mathrm{min}^{\mathrm{Thm}}{ }^{\prime}} \leq C^{\prime}$. By $N$-protoalgebraicity of $\ell$ and the surjectivity of $\langle F, \alpha\rangle$, we obtain, using Proposition 3.11 of [24], that $\Omega^{N^{\prime}}\left(\mathrm{Thm}^{\prime}\right)=\widetilde{\Omega}^{N^{\prime}}\left(C^{\prime}\right)$. Now, for any theory family $T^{\prime}$ of $C^{\prime \mathrm{min}^{\mathrm{Thm}}}$, we have that $\mathrm{Thm}^{\prime} \leq T^{\prime}$, whence, by $N^{\prime}$-protoalgebraicity, $\Omega^{N^{\prime}}\left(\mathrm{Thm}^{\prime}\right) \leq \Omega^{N^{\prime}}\left(T^{\prime}\right)$. Thus, $\Omega^{N^{\prime}}\left(\mathrm{Thmm}^{\prime}\right)=\widetilde{\Omega}^{N^{\prime}}\left(C^{\prime}\right)$ is compatible with $T^{\prime}$ which gives that $T^{\prime}$ is a theory family of $C^{\prime}$ and therefore $C^{\prime}=C^{\prime \mathrm{min}^{\mathrm{Thm}}}$.

Motivated by the definition of the subclass $\mathrm{Fi}_{\S}^{\star} \mathrm{A}$ of those logical filters $F$ that generate principal lattice filters in the lattice of $\delta$-filters of an algebra $\mathbf{A}$ that form the closed set systems of full models of $\delta$ on $\mathbf{A}$ and by the Isomorphism Theorem 2.30 of [13], which was adapted to the $\pi$-institution framework in [30], we give the following definition of a similar subclass of the class of all theory systems of a model of a $\pi$-institution $\ell$. Suppose $\ell=\langle\operatorname{Sign}, \mathrm{SEN}, C\rangle$ is a $\pi$-institution with $N$ a category of natural transformations on SEN. Let $\mathrm{SEN}^{\prime}:$ Sign' $^{\prime} \rightarrow$ Set be a functor, $N^{\prime}$ a category of natural transformations on $\mathrm{SEN}^{\prime}$, and $\langle F, \alpha\rangle: \mathrm{SEN} \rightarrow{ }^{\text {se }} \mathrm{SEN}^{\prime}$ a surjective $\left(N, N^{\prime}\right)$-epimorphic translation. Define

$$
\operatorname{ThSys}_{\ell}^{\left\langle F, \alpha{ }^{\star}\right.}\left(\mathrm{SEN}^{\prime}\right)
$$

to be the collection of all those theory systems $T^{\prime}$ of the $\langle F, \alpha\rangle$-min $\left(N, N^{\prime}\right)$-model $\ell^{\prime \text { min }}=\left\langle\right.$ Sign $\left.^{\prime}, \mathrm{SEN}^{\prime}, C^{\prime \text { min }}\right\rangle$ of $\ell$ on $\mathrm{SEN}^{\prime}$ such that $\ell^{\prime \min ^{T^{\prime}}}=\left\langle\mathbf{S i g n}^{\prime}, \mathrm{SEN}^{\prime}, C^{\prime \min ^{T^{\prime}}}\right\rangle$ is an $\langle F, \alpha\rangle$-full $\left(N, N^{\prime}\right)$-model of $\ell$.

Proposition 5.3 Suppose $\ell=\langle$ Sign, SEN, $C\rangle$ is a $\pi$-institution with $N$ a category of natural transformations on SEN. Let $\mathrm{SEN}^{\prime}:$ Sign' $^{\rightarrow}$ Set be a functor, $N^{\prime}$ a category of natural transformations on $\mathrm{SEN}^{\prime}$, and $\langle F, \alpha\rangle: \mathrm{SEN} \rightarrow{ }^{\text {se }} \mathrm{SEN}^{\prime}$ a surjective $\left(N, N^{\prime}\right)$-epimorphic translation. If $\ell$ is $N$-protoalgebraic, then the Leibniz operator $\Omega_{\mathrm{SEN}^{\prime}}^{\langle F, \alpha\rangle}:=\Omega^{N^{\prime}}$ is a lattice isomorphism from $\operatorname{ThSys}_{\ell}^{\langle F, \alpha\rangle}{ }^{\star}\left(\mathrm{SEN}^{\prime}\right)$ to $\operatorname{Con}_{\operatorname{Alg}^{N}(\ell)}^{\langle F, \alpha\rangle}\left(\operatorname{SEN}^{\prime}\right)$.

Proof Denote by $\ell^{\prime \text { min }}=\left\langle\mathbf{S i g n}^{\prime}, \mathrm{SEN}^{\prime}, C^{\prime \text { min }}\right\rangle$ the $\langle F, \alpha\rangle$-min $\left(N, N^{\prime}\right)$-model of $\ell$ on $\mathrm{SEN}^{\prime}$. Given a theory system $T$ in $\mathrm{ThSys}_{\ell}^{\langle F, \alpha\rangle}{ }^{\star}\left(\mathrm{SEN}^{\prime}\right)$, the $\pi$-institution $\ell^{\prime \min ^{T}}=\left\langle\mathbf{S i g n}^{\prime}, \mathrm{SEN}^{\prime}, C^{\prime \min ^{T}}\right\rangle$ is by definition an $\langle F, \alpha\rangle$-full $\left(N, N^{\prime}\right)$-model of 


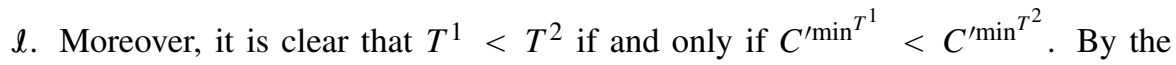
$N$-protoalgebraicity of $\ell$ and the surjectivity of $\langle F, \alpha\rangle$, we obtain, using Theorem 5.2, that every $\langle F, \alpha\rangle$-full $\left(N, N^{\prime}\right)$-model of $\ell$ is of the form $\ell^{\prime \min ^{T}}$ for some theory system $T$ in $\mathrm{ThSys}_{\ell}^{\langle F, \alpha\rangle}\left(\mathrm{SEN}^{\prime}\right)$. This proves that $T \mapsto C^{\prime \mathrm{min}^{T}}$ is an order isomorphism between the collection of theory systems in $\operatorname{ThSys}_{\ell}^{\langle F, \alpha\rangle^{\star}}\left(\operatorname{SEN}^{\prime}\right)$ and the full models in $\operatorname{FMod}_{\ell}^{\langle F, \alpha\rangle}\left(\mathrm{SEN}^{\prime}\right)$. Now, applying Theorem 13 of [30], we get an order-isomorphism from $\mathbf{T h S y s}_{\ell}^{\langle F, \alpha\rangle}\left(\mathrm{SEN}^{\prime}\right)$ to $\operatorname{Con}_{\operatorname{Ag}^{N}(\ell)}^{\langle F, \alpha\rangle}\left(\operatorname{SEN}^{\prime}\right)$. The $N$ protoalgebraicity of $\ell$ and the surjectivity of $\langle F, \alpha\rangle$ combined with Proposition 3.11 of [24] now yield that the mapping is the Leibniz operator $\Omega^{N^{\prime}}$ applied to the theory systems of the $\langle F, \alpha\rangle$-min $\left(N, N^{\prime}\right)$-model $\ell^{\prime \min }$ of $\ell$ on $\mathrm{SEN}^{\prime}$.

Following [13], we define the relation $\sim$ between two theory systems $T^{1}, T^{2}$ in the collection $\mathrm{ThSys}_{\ell}^{\langle F, \alpha\rangle}\left(\mathrm{SEN}^{\prime}\right)$ of all theory systems of the $\langle F, \alpha\rangle$-min $\left(N, N^{\prime}\right)$-model of $\ell$ on $\mathrm{SEN}^{\prime}$ by

$$
T^{1} \sim T^{2} \quad \text { iff } \quad \Omega^{N^{\prime}}\left(T^{1}\right)=\Omega^{N^{\prime}}\left(T^{2}\right)
$$

that is, $\sim$ is the kernel of the $N^{\prime}$-Leibniz operator as applied on the collection $\mathrm{ThSys}_{\ell}^{\langle F, \alpha\rangle}\left(\mathrm{SEN}^{\prime}\right)$. Proposition 5.3 shows that, for $\ell N$-protoalgebraic, at most one of the theory systems in each $\sim$-equivalence class is a member of $\mathrm{ThSys}_{\ell}^{\langle F, \alpha\rangle^{\star}}\left(\mathrm{SEN}^{\prime}\right)$. We prove an analog of Proposition 3.6 of [13] that characterizes that theory system.

If $\ell$ is $N$-protoalgebraic and $\langle F, \alpha\rangle: \operatorname{SEN} \rightarrow^{\text {se }} \operatorname{SEN}^{\prime}$ is a surjective $\left(N, N^{\prime}\right)$ epimorphic translation, then, denoting by $T / \sim$ the $\sim$-equivalence class of a theory family $T$ of the $\langle F, \alpha\rangle$-min $\left(N, N^{\prime}\right)$-model $\ell^{\prime \text { min }}$ of $\ell$ on $\mathrm{SEN}^{\prime}$, we get that

$$
\Omega^{N^{\prime}}(\bigcap T / \sim)=\bigcap_{T^{\prime} \in T / \sim} \Omega^{N^{\prime}}\left(T^{\prime}\right)=\bigcap_{T^{\prime} \in T / \sim} \Omega^{N^{\prime}}(T)=\Omega^{N^{\prime}}(T)
$$

whence the theory system $\bigcap T / \sim$ is in the same $\sim$-class with $T$. We show that $\bigcap T / \sim$ is in $\operatorname{ThSys}_{\ell}^{\langle F, \alpha\rangle^{\star}}\left(\mathrm{SEN}^{\prime}\right)$, which will prove that that theory system is the representative of $T / \sim$ in $\operatorname{ThSys}_{\ell}^{\langle F, \alpha\rangle}\left(\operatorname{SEN}^{\prime}\right)$.

Proposition 5.4 Suppose $\ell=\langle$ Sign, SEN, $C\rangle$ is a $\pi$-institution and $N$ a category of natural transformations on SEN. Let $\mathrm{SEN}^{\prime}:$ Sign' $\rightarrow$ Set be a functor, $N^{\prime}$ a category of natural transformations on $\mathrm{SEN}^{\prime}$, and $\langle F, \alpha\rangle: \mathrm{SEN} \rightarrow{ }^{\text {se }} \mathrm{SEN}^{\prime}$ a surjective $\left(N, N^{\prime}\right)$-epimorphic translation. If $\ell$ is $N$-protoalgebraic and $T$ is a theory system of the $\langle F, \alpha\rangle$-min $\left(N, N^{\prime}\right)$-model of $\ell$ on $\mathrm{SEN}^{\prime}$, then the following statements are equivalent:

1. $T \in \operatorname{ThSys}_{\ell}^{\langle F, \alpha\rangle^{\star}}\left(\mathrm{SEN}^{\prime}\right)$;

2. $T$ is the least element in the class $T / \sim$;

3. $T / \Omega^{N^{\prime}}(T)$ is the least element in $\mathrm{ThSys}_{\ell}^{\left\langle F, \pi_{F}^{\Omega^{N^{\prime}}(T)} \alpha\right\rangle}\left(\operatorname{SEN}^{\Omega^{N^{\prime}}(T)}\right)$. 


\section{Proof}

$2 \rightarrow 3$ Suppose that the theory system $T$ is the least element in the class $T / \sim$ and consider $\left.T^{\prime \prime} \in \operatorname{ThSys}_{\ell}^{\left\langle F, \pi_{F}^{\Omega^{N^{\prime}}}(T)\right.} \alpha\right\rangle\left(\operatorname{SEN}^{\prime \Omega^{N^{\prime}}(T)}\right)$. Let

$$
T^{\prime}=\left(\pi^{\Omega^{N^{\prime}}(T)}\right)^{-1}\left(T^{\prime \prime}\right) \cap T \in \operatorname{ThSys}_{\ell}^{\langle F, \alpha\rangle}\left(\mathrm{SEN}^{\prime}\right) .
$$

Then we have that

$$
\begin{aligned}
T^{\prime} & =\left(\pi^{\Omega^{N^{\prime}}(T)}\right)^{-1}\left(T^{\prime \prime}\right) \cap T \\
& =\left(\pi^{\Omega^{N^{\prime}}(T)}\right)^{-1}\left(T^{\prime \prime}\right) \cap\left(\pi^{\Omega^{N^{\prime}}}(T)\right)^{-1}\left(\pi^{\Omega^{N^{\prime}}(T)}(T)\right) \\
& =\left(\pi^{\Omega^{N^{\prime}}(T)}\right)^{-1}\left(T^{\prime \prime} \cap \pi^{\Omega^{N^{\prime}}(T)}(T)\right),
\end{aligned}
$$

which shows that $T^{\prime}$ is a union of equivalence classes of $\Omega^{N^{\prime}}(T)$, whence $\Omega^{N^{\prime}}(T)$ is compatible with $T^{\prime}$. Thus, by the definition of the Leibniz congruence system, $\Omega^{N^{\prime}}(T) \leq \Omega^{N^{\prime}}\left(T^{\prime}\right)$.

Note, on the other hand, that by its definition $T^{\prime} \leq T$, whence, since $\ell$ is $N$-protoalgebraic and $\langle F, \alpha\rangle$ is surjective, we have by Proposition 5.27 of [24] that $\ell^{\prime}$ is $N^{\prime}$-protoalgebraic and therefore that $\Omega^{N^{\prime}}\left(T^{\prime}\right) \leq \Omega^{N^{\prime}}(T)$, and this establishes that $\Omega^{N^{\prime}}\left(T^{\prime}\right)=\Omega^{N^{\prime}}(T)$; that is, $T^{\prime} \sim T$. Since by hypothesis $T$ is the least element in $T / \sim$, we have that $T \leq T^{\prime}$ which combined with the definition of $T^{\prime}$ yields that $T^{\prime}=T$. Therefore $T \leq\left(\pi^{\Omega^{N^{\prime}}(T)}\right)^{-1}\left(T^{\prime \prime}\right)$ and, hence, $T / \Omega^{N^{\prime}}(T)=\pi^{\Omega^{N^{\prime}}(T)}(T) \leq T^{\prime \prime}$. This shows that $T / \Omega^{N^{\prime}}(T)$ is indeed the least element in $\operatorname{ThSys}_{\ell}^{\left\langle F, \pi_{F}^{\Omega^{N^{\prime}}(T)} \alpha\right\rangle}\left(\operatorname{SEN}^{\prime \Omega^{N^{\prime}}(T)}\right)$.

$3 \rightarrow 1$ Since $\ell$ is $N$-protoalgebraic and $\langle F, \alpha\rangle$ is surjective, we obtain, by Proposition 5.27 of [24], that $\ell^{\prime}$ is $N^{\prime}$-protoalgebraic. Now, since $\ell^{\prime}$ is $N^{\prime}$ protolagebraic, we obtain that the natural projection $T^{\prime} \mapsto T^{\prime} / \Omega^{N^{\prime}}(T)$ is a lattice isomorphism between the theory families of $\ell^{\prime}$ containing $T$ and the lattice of all theory families of $\ell^{\prime T} / \Omega^{N^{\prime}}(T)$. Using the hypothesis, we obtain that the lattice of all theory families of $\ell^{\prime T} / \Omega^{N^{\prime}}(T)$ is identical to the lattice of all theory families of the $\left\langle F, \pi_{F}^{\Omega^{N^{\prime}}(T)} \alpha\right\rangle$-min $\left(N, N^{\prime \Omega^{N^{\prime}}(T)}\right)$-model of $\ell$ on $\operatorname{SEN}^{\prime \Omega^{N^{\prime}}(T)}$. Taking into account that by $N^{\prime}$-protoalgebraicity $\widetilde{\Omega}^{N^{\prime}}\left(\ell^{\prime \min ^{T}}\right)=\Omega^{N^{\prime}}(T)$, we have that $\ell^{\prime \min ^{T}}=\left\langle\mathbf{S i g n}^{\prime}, \mathrm{SEN}^{\prime}, C^{\prime \min ^{T}}\right\rangle$ is the $\langle F, \alpha\rangle$-min $\left(N, N^{\prime}\right)$-model of $\ell$. This yields that the theory system $T \in \operatorname{ThSys}_{\ell}^{\langle F, \alpha\rangle^{\star}}\left(\operatorname{SEN}^{\prime}\right)$.

$\mathbf{1} \rightarrow \mathbf{2}$ Suppose that $T \in \mathrm{ThSys}_{\ell}^{\langle F, \alpha\rangle}{ }^{\star}\left(\operatorname{SEN}^{\prime}\right)$. Since $\ell$ is $N$-protoalgebraic and $\langle F, \alpha\rangle$ is surjective, $\ell^{\prime}$ is $N^{\prime}$-protoalgebraic, by Proposition 5.27 of [24], whence there exists a $\leq$-least $T^{\prime} \in \mathrm{ThSys}_{\ell}^{\langle F, \alpha\rangle}\left(\mathrm{SEN}^{\prime}\right)$ such that $\Omega^{N^{\prime}}\left(T^{\prime}\right)=\Omega^{N^{\prime}}(T)$. If we now combine $2 \rightarrow 3 \rightarrow 1$ above, we obtain that $T^{\prime} \in \operatorname{ThSys}_{\ell}^{\langle F, \alpha\rangle}{ }^{\star}\left(\operatorname{SEN}^{\prime}\right)$; that is, $\ell^{\prime \min ^{T^{\prime}}}$ is a full $\left(N, N^{\prime}\right)$-model of $\ell$ on $\operatorname{SEN}^{\prime}$ via $\langle F, \alpha\rangle$. By assumption, it is also the case that $\ell^{\prime \mathrm{min}^{T}}$ is a full $\left(N, N^{\prime}\right)$-model of $\ell$ on $\mathrm{SEN}^{\prime}$ via $\langle F, \alpha\rangle$. Therefore, by 
$N^{\prime}$-protoalgebraicity,

$$
\begin{aligned}
\widetilde{\Omega}^{N^{\prime}}\left(\ell^{\min ^{T^{\prime}}}\right) & =\Omega^{N^{\prime}}\left(T^{\prime}\right) \\
& =\Omega^{N^{\prime}}(T) \\
& =\widetilde{\Omega}^{N^{\prime}}\left(\ell^{\prime \min ^{T}}\right) .
\end{aligned}
$$

Now by Theorem 13 of [30], we obtain that $\ell^{\min ^{T^{\prime}}}=\ell^{\min ^{T}}$; that is, $T=T^{\prime}$. Hence $T$ is indeed the $\leq-$-least theory system in its $\sim$-equivalence class.

As a corollary, we obtain an analog of Proposition 3.7 of [13] which will serve as the starting point of the study of weakly algebraizable $\pi$-institutions in a forthcoming sequel to the present paper.

Proposition 5.5 Suppose $\ell=\langle$ Sign, SEN, $C\rangle$ is a $\pi$-institution with $N$ a category of natural transformations on SEN. Let $\mathrm{SEN}^{\prime}: \mathbf{S i g n}^{\prime} \rightarrow$ Set be a functor, $N^{\prime}$ a category of natural transformations on $\mathrm{SEN}^{\prime}$, and $\langle F, \alpha\rangle: \mathrm{SEN} \rightarrow{ }^{\text {se }} \mathrm{SEN}^{\prime}$ a surjective $\left(N, N^{\prime}\right)$-epimorphic translation. If $\ell$ is $N$-protoalgebraic, then $\operatorname{ThSys}_{\ell}^{\langle F, \alpha\rangle^{\star}}\left(\mathrm{SEN}^{\prime}\right)=\operatorname{ThSys}_{\ell}^{\langle F, \alpha\rangle}\left(\operatorname{SEN}^{\prime}\right)$ if and only if $\Omega_{\mathrm{SEN}^{\prime}}^{\langle F, \alpha\rangle}$ is injective on $\operatorname{ThSys}_{\ell}^{\langle F, \alpha\rangle}\left(\mathrm{SEN}^{\prime}\right)$.

Proof $\operatorname{ThSys}_{\ell}^{\langle F, \alpha\rangle}\left(\mathrm{SEN}^{\prime}\right)=\operatorname{ThSys}_{\ell}^{\langle F, \alpha\rangle}\left(\mathrm{SEN}^{\prime}\right)$ if and only if every theory system $T$ of the $\langle F, \alpha\rangle$-min model of $\ell$ on SEN' is the only member in $T / \sim$ if and only if $\Omega_{\mathrm{SEN}^{\prime}}^{\langle F, \alpha\rangle}$ is injective on $\mathrm{ThSys}_{\ell}^{\langle F, \alpha\rangle}\left(\mathrm{SEN}^{\prime}\right)$.

\section{A Lindenbaum-Tarski Counterpart}

In this last section of the paper, an effort is made to reconcile some aspects of the syntactic approach to the algebraic study of $\pi$-institutions with some aspects of the semantic approach. The syntactic approach in the context of algebraizability refers to the existence of some syntactic entities inside the $\pi$-institution such as implication systems or parameterized equivalence systems as well as the existence of appropriate translations, semi-interpretations, or interpretations allowing interactions between the $\pi$-institution and its models or its algebraiclike counterparts. The semantic aspects comprise all features and properties that are based on the study of the $\pi$-institution using the operator approach, for example, its classification via the Leibniz or the Tarski operators into the steps of the abstract algebraic hierarchy for $\pi$-institutions. In this section, an algebraiclike counterpart $\mathcal{L}^{N}(\ell)$ of a given $\pi$-institution $\ell=\langle$ Sign, SEN, $C\rangle$, with $N$ a category of natural transformations on SEN, is constructed, called the $N$-Lindenbaum-Tarski counterpart of $\ell$, and it is shown that there exists a way to automatically translate equations of this algebraiclike counterpart into sentences of $\ell$ via what is called the Blok-Pigozzi mapping of $\ell$. If this mapping (which unfortunately is not a translation) satisfies an additional technical property, called the $(\beta, N)$-translation property, in $\ell$, then it is shown that it may be used to characterize protoalgebraicity.

Let $\ell=\langle$ Sign, SEN, $C\rangle$ be a $\pi$-institution with $N$ a category of natural transformations on SEN. An $n$-ary function symbol $\sigma^{\#}$ is associated to every natural transformation $\sigma: \operatorname{SEN}^{n} \rightarrow \operatorname{SEN}$ in $N$. Then, the quadruple $\mathcal{L}^{N}(\ell)=\langle$ Sign, EQ, ALG, $\models\rangle$ is defined as follows: 
1 View $N^{\#}=\left\{\sigma^{\#}: \sigma: \operatorname{SEN}^{n} \rightarrow\right.$ SEN in $\left.N\right\}$ as an algebraic signature and set, for all $\Sigma \in|\mathbf{S i g n}|$,

$$
\mathrm{EQ}(\Sigma)=\operatorname{Tm}_{N^{\#}}(\operatorname{SEN}(\Sigma))^{2},
$$

that is, the set of all pairs of $N^{\#}$-terms built up in the usual recursive way using the elements of $\operatorname{SEN}(\Sigma)$ in the role of variables. Furthermore, for all $\Sigma_{1}, \Sigma_{2} \in|\operatorname{Sign}|$, $f \in \operatorname{Sign}\left(\Sigma_{1}, \Sigma_{2}\right)$, define $\operatorname{EQ}(f): \operatorname{Tm}_{N^{\#}}\left(\operatorname{SEN}\left(\Sigma_{1}\right)\right)^{2} \rightarrow \operatorname{Tm}_{N^{\#}}\left(\operatorname{SEN}\left(\Sigma_{2}\right)\right)^{2}$ to be the Cartesian square of the map $f^{*}: \operatorname{Tm}_{N^{*}}\left(\operatorname{SEN}\left(\Sigma_{1}\right)\right) \rightarrow \operatorname{Tm}_{N^{\#}}\left(\operatorname{SEN}\left(\Sigma_{2}\right)\right)$ that is defined by recursion on the structure of an $N^{\#}$-term in $\operatorname{SEN}\left(\Sigma_{1}\right)$ as follows:

(a) $f^{*}(\varphi)=\operatorname{SEN}(f)(\varphi)$, for all $\varphi \in \operatorname{SEN}\left(\Sigma_{1}\right)$, and

(b) $f^{*}\left(\sigma^{\#}\left(t_{0}, \ldots, t_{n-1}\right)\right)=\sigma^{\#}\left(f^{*}\left(t_{0}\right), \ldots, f^{*}\left(t_{n-1}\right)\right)$, for all $\sigma^{\#} n$-ary and all $t_{0}, \ldots, t_{n-1} \in \operatorname{Tm}_{N^{*}}\left(\operatorname{SEN}\left(\Sigma_{1}\right)\right)$.

2 For every $\Sigma \in|\mathbf{S i g n}|$, let $\operatorname{ALG}(\Sigma)$ be the category with objects triples of the form $\left\langle\left\langle\operatorname{SEN}^{\Omega^{N}(T)}\left(\Sigma^{\prime}\right),\left\{\sigma_{\Sigma^{\prime}}^{\Omega^{N}(T)}\right\}\right\rangle, f\right\rangle, \Sigma^{\prime} \in|\operatorname{Sign}|, f \in \operatorname{Sign}\left(\Sigma, \Sigma^{\prime}\right), T \in \operatorname{ThFam}(\ell)$, where

(a) $\operatorname{SEN}^{\Omega^{N}(T)}\left(\Sigma^{\prime}\right)=\operatorname{SEN}\left(\Sigma^{\prime}\right) / \Omega_{\Sigma^{\prime}}^{N}(T)$ and

(b) $\sigma^{\Omega^{N}(T)}$ is the natural transformation on $\operatorname{SEN}^{\Omega^{N}(T)}$ induced by the natural transformation $\sigma: \mathrm{SEN}^{n} \rightarrow \operatorname{SEN}$ in $N$ and $\left\{\sigma_{\Sigma^{\prime}}^{\Omega^{N}(T)}\right\}:=\left\{\sigma_{\Sigma^{\prime}}^{\Omega^{N}(T)}: \sigma\right.$ in $\left.N\right\}$. and morphisms

$$
h:\left\langle\left\langle\operatorname{SEN}^{\Omega^{N}\left(T^{\prime}\right)}\left(\Sigma^{\prime}\right),\left\{\sigma_{\Sigma^{\prime}}^{\Omega^{N}\left(T^{\prime}\right)}\right\}\right\rangle, f\right\rangle \rightarrow\left\langle\left\langle\operatorname{SEN}^{\Omega^{N}\left(T^{\prime \prime}\right)}\left(\Sigma^{\prime \prime}\right),\left\{\sigma_{\Sigma^{\prime \prime}}^{\Omega^{N}\left(T^{\prime \prime}\right)}\right\}\right\rangle, g\right\rangle
$$

all universal algebraic $N^{\#}$-homomorphisms

$$
h:\left\langle\operatorname{SEN}^{\Omega^{N}\left(T^{\prime}\right)}\left(\Sigma^{\prime}\right),\left\{\sigma_{\Sigma^{\prime}}^{\Omega^{N}\left(T^{\prime}\right)}\right\}\right\rangle \rightarrow\left\langle\operatorname{SEN}^{\Omega^{N}\left(T^{\prime \prime}\right)}\left(\Sigma^{\prime \prime}\right),\left\{\sigma_{\Sigma^{\prime \prime}}^{\Omega^{N}\left(T^{\prime \prime}\right)}\right\}\right\rangle
$$

such that the following triangle commutes:

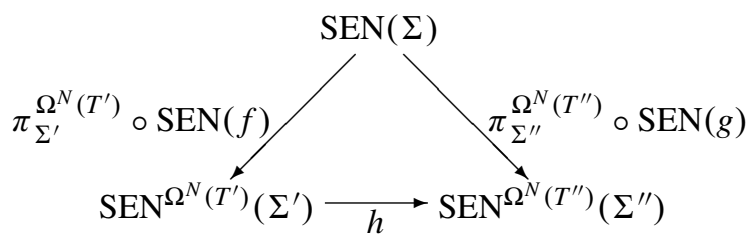

At the morphism level, given a morphism $k \in \operatorname{Sign}\left(\Sigma_{1}, \Sigma_{2}\right)$, the functor $\operatorname{ALG}(k)$ : $\operatorname{ALG}\left(\Sigma_{2}\right) \rightarrow \operatorname{ALG}\left(\Sigma_{1}\right)$ maps an object $\left\langle\left\langle\operatorname{SEN}^{\Omega^{N}(T)}\left(\Sigma^{\prime}\right), \quad\left\{\sigma_{\Sigma^{\prime}}^{\Omega^{N}(T)}\right\}\right\rangle, f\right\rangle$ $\in\left|\operatorname{ALG}\left(\Sigma_{2}\right)\right|$ to

$$
\operatorname{ALG}(k)\left(\left\langle\left\langle\operatorname{SEN}^{\Omega^{N}(T)}\left(\Sigma^{\prime}\right),\left\{\sigma_{\Sigma^{\prime}}^{\Omega^{N}(T)}\right\}\right\rangle, f\right\rangle\right)=\left\langle\left\langle\operatorname{SEN}^{\Omega^{N}(T)}\left(\Sigma^{\prime}\right),\left\{\sigma_{\Sigma^{\prime}}^{\Omega^{N}(T)}\right\}\right\rangle, f k\right\rangle
$$

and a morphism

$$
h:\left\langle\left\langle\operatorname{SEN}^{\Omega^{N}\left(T^{\prime}\right)}\left(\Sigma^{\prime}\right),\left\{\sigma_{\Sigma^{\prime}}^{\Omega^{N}\left(T^{\prime}\right)}\right\}\right\rangle, f\right\rangle \rightarrow\left\langle\left\langle\operatorname{SEN}^{\Omega^{N}\left(T^{\prime \prime}\right)}\left(\Sigma^{\prime \prime}\right),\left\{\sigma_{\Sigma^{\prime \prime}}^{\Omega^{N}\left(T^{\prime \prime}\right)}\right\}\right\rangle, g\right\rangle
$$

to the morphism

$$
\begin{aligned}
& \operatorname{ALG}(k)(h)=\begin{array}{l}
h:\left\langle\left\langle\operatorname{SEN}^{\Omega^{N}\left(T^{\prime}\right)}\left(\Sigma^{\prime}\right),\left\{\sigma_{\Sigma^{\prime}}^{\Omega^{N}\left(T^{\prime}\right)}\right\}\right\rangle, f k\right\rangle \rightarrow \\
\quad\left\langle\left\langle\operatorname{SEN}^{\Omega^{N}\left(T^{\prime \prime}\right)}\left(\Sigma^{\prime \prime}\right),\left\{\sigma_{\Sigma^{\prime \prime}}^{\Omega^{N}}\left(T^{\prime \prime}\right)\right\}\right\rangle, g k\right\rangle .
\end{array}
\end{aligned}
$$


3 Finally, for every $\Sigma \in|\mathbf{S i g n}|$, the $\Sigma$-satisfaction

$$
\models_{\Sigma} \subseteq|\mathrm{ALG}(\Sigma)| \times \mathrm{EQ}(\Sigma)
$$

is defined, for all $\left\langle\left\langle\operatorname{SEN}^{\Omega^{N}(T)}\left(\Sigma^{\prime}\right),\left\{\sigma_{\Sigma^{\prime}}^{\Omega^{N}(T)}\right\}\right\rangle, f\right\rangle \in|\operatorname{ALG}(\Sigma)|,\left\langle t_{0}, t_{1}\right\rangle \in \mathrm{EQ}(\Sigma)$, by

$$
\begin{gathered}
\left\langle\left\langle\operatorname{SEN}^{\Omega^{N}(T)}\left(\Sigma^{\prime}\right),\left\{\sigma_{\Sigma^{\prime}}^{\Omega^{N}(T)}\right\}\right\rangle, f\right\rangle \models \Sigma\left\langle t_{0}, t_{1}\right\rangle \quad \text { iff } \\
\operatorname{Eval}^{\left\langle\operatorname{SEN}^{\Omega^{N}(T)}\left(\Sigma^{\prime}\right),\left\{\sigma_{\Sigma^{\prime}}^{\Omega^{N}(T)}\right\}\right\rangle}\left(f^{*}\left(t_{0}\right)\right)= \\
\operatorname{Eval}^{\left\langle\operatorname{SEN}^{\Omega^{N}(T)}\left(\Sigma^{\prime}\right),\left\{\sigma_{\Sigma^{\prime}}^{\Omega^{N}(T)}\right\}\right\rangle}\left(f^{*}\left(t_{1}\right)\right),
\end{gathered}
$$

where Eval ${ }^{\left\langle\operatorname{SEN}^{\Omega^{N}(T)}\left(\Sigma^{\prime}\right),\left\{\sigma_{\Sigma^{\prime}}^{\Omega^{N}(T)}\right\}\right\rangle}: \operatorname{Tm}_{N^{\#}}\left(\operatorname{SEN}\left(\Sigma^{\prime}\right)\right) \rightarrow \operatorname{SEN}^{\Omega^{N}(T)}\left(\Sigma^{\prime}\right)$ is the function that evaluates $N^{\#}$-terms into the $N^{\#}$-algebra

$$
\left\langle\operatorname{SEN}^{\Omega^{N}(T)}\left(\Sigma^{\prime}\right),\left\{\sigma_{\Sigma^{\prime}}^{\Omega^{N}(T)}\right\}\right\rangle
$$

under the interpretation $\varphi \mapsto \varphi^{\Omega^{N}(T)}$, for all $\varphi \in \operatorname{SEN}\left(\Sigma^{\prime}\right)$ (in the ordinary universal algebraic sense).

Some details on the rigorous precise definition of

$$
\operatorname{Eval}^{\left\langle\operatorname{SEN}^{\Omega^{N}(T)}(\Sigma),\left\{\sigma_{\Sigma}^{\Omega^{N}(T)}\right\}\right\rangle}: \operatorname{Tm}_{N^{\#}}(\operatorname{SEN}(\Sigma)) \rightarrow \operatorname{SEN}^{\Omega^{N}(T)}(\Sigma)
$$

are provided now, since the need will arise for its formal use: It is given by recursion on the structure of $N^{\#}$-terms in $\operatorname{SEN}(\Sigma)$. The base case of the recursion is provided by

$$
\operatorname{Eval}^{\left\langle\operatorname{SEN}^{\Omega^{N}(T)}(\Sigma),\left\{\sigma_{\Sigma}^{\Omega^{N}(T)}\right\}\right\rangle}(\varphi)=\varphi^{\Omega^{N}(T)} \text {, for all } \varphi \in \operatorname{SEN}(\Sigma) .
$$

For the recursion step, suppose that $t=\sigma^{\#}\left(t_{0}, \ldots, t_{n-1}\right)$ and that the values $\mathrm{Eval}^{\left\langle\operatorname{SEN}^{\Omega^{N}(T)}(\Sigma),\left\{\sigma_{\Sigma}^{\Omega^{N}(T)}\right\}\right\rangle}\left(t_{i}\right)$ have already been defined, for all $i=0, \ldots, n-1$. Then

$$
\begin{aligned}
& \operatorname{Eval}^{\left\langle\operatorname{SEN}^{\Omega^{N}(T)}(\Sigma),\left\{\sigma_{\Sigma}^{\Omega^{N}(T)}\right\}\right\rangle}\left(\sigma^{\#}\left(t_{0}, \ldots, t_{n-1}\right)\right)= \\
& \sigma_{\Sigma}^{\Omega^{N}(T)}\left(\operatorname{Eval}^{\left\langle\operatorname{SEN}^{\Omega^{N}(T)}(\Sigma),\left\{\sigma_{\Sigma}^{\Omega^{N}(T)}\right\}\right\rangle}\left(t_{0}\right), \ldots,\right. \\
& \left.\operatorname{Eval}^{\left\langle\operatorname{SEN}^{\Omega^{N}(T)}(\Sigma),\left\{\sigma_{\Sigma}^{\Omega^{N}(T)}\right\}\right\rangle}\left(t_{n-1}\right)\right) .
\end{aligned}
$$

To avoid the cumbersome notation we set

$$
\operatorname{Eval}^{\langle N, T, \Sigma\rangle}:=\operatorname{Eval}^{\left\langle\operatorname{SEN}^{\Omega^{N}(T)}(\Sigma),\left\{\sigma_{\Sigma}^{\Omega^{N}(T)}\right\}\right\rangle} .
$$

To show that the construction just introduced gives in fact an institution, the following technical lemma is needed.

Lemma 6.1 Let $\ell=\langle$ Sign, SEN, $C\rangle$ be a $\pi$-institution and $N$ a category of natural transformations on SEN. Then, for all $\Sigma_{1}, \Sigma_{2}, \Sigma^{\prime} \in|\operatorname{Sign}|, f \in \operatorname{Sign}\left(\Sigma_{2}, \Sigma^{\prime}\right), k$ $\in \operatorname{Sign}\left(\Sigma_{1}, \Sigma_{2}\right)$, and all $t \in \operatorname{Tm}_{N^{\#}}\left(\operatorname{SEN}\left(\Sigma_{1}\right)\right)$,

$$
(f k)^{*}(t)=f^{*}\left(k^{*}(t)\right)
$$




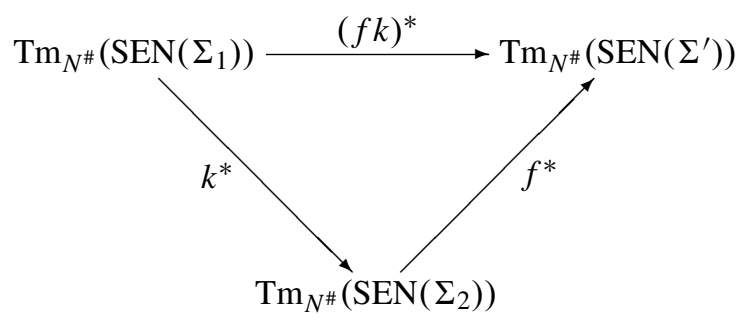

Proof The proof is by induction on the structure of the $N^{\#}$-term $t$ in $\operatorname{SEN}\left(\Sigma_{1}\right)$. If $\varphi \in \operatorname{SEN}\left(\Sigma_{1}\right)$, then we have that

$$
\begin{aligned}
(f k)^{*}(\varphi) & =\operatorname{SEN}(f k)(\varphi) \\
& =\operatorname{SEN}(f)(\operatorname{SEN}(k)(\varphi)) \\
& =\operatorname{SEN}(f)\left(k^{*}(\varphi)\right) \\
& =f^{*}\left(k^{*}(\varphi)\right) .
\end{aligned}
$$

Finally, if $\sigma^{\#}$ is $n$-ary and $t_{0}, \ldots, t_{n-1} \in \operatorname{Tm}_{N^{\#}}\left(\operatorname{SEN}\left(\Sigma_{1}\right)\right)$,

$$
\begin{aligned}
(f k)^{*}\left(\sigma^{\#}\left(t_{0}, \ldots, t_{n-1}\right)\right) & =\sigma^{\#}\left((f k)^{*}\left(t_{0}\right), \ldots,(f k)^{*}\left(t_{n-1}\right)\right) \\
& =\sigma^{\#}\left(f^{*}\left(k^{*}\left(t_{0}\right)\right), \ldots, f^{*}\left(k^{*}\left(t_{n-1}\right)\right)\right) \\
& =f^{*}\left(\sigma^{\#}\left(k^{*}\left(t_{0}\right), \ldots, k^{*}\left(t_{n-1}\right)\right)\right. \\
& =f^{*}\left(k^{*}\left(\sigma^{\#}\left(t_{0}, \ldots, t_{n-1}\right)\right)\right) .
\end{aligned}
$$

Proposition 6.2 If $\ell=\langle\operatorname{Sign}, \mathrm{SEN}, C\rangle$ is a $\pi$-institution with $N$ a category of natural transformations on $\mathrm{SEN}$, then $\mathcal{L}^{N}(\ell)=\langle$ Sign, EQ, MOD, $\models\rangle$ is an institution.

Proof It is not difficult to verify that EQ : Sign $\rightarrow$ Set is a functor and that ALG : Set $\rightarrow$ CAT $^{\text {op }}$ is also a functor. We only verify the satisfaction condition in detail here. To this end, let $\Sigma_{1}, \Sigma_{2} \in|\operatorname{Sign}|, k \in \operatorname{Sign}\left(\Sigma_{1}, \Sigma_{2}\right),\left\langle\left\langle\operatorname{SEN}^{\Omega^{N}}(T)\left(\Sigma^{\prime}\right)\right.\right.$, $\left.\left.\left\{\sigma_{\Sigma^{\prime}}^{\Omega^{N}(T)}\right\}\right\rangle, f\right\rangle \in\left|\operatorname{ALG}\left(\Sigma_{2}\right)\right|$, and $\left\langle t_{0}, t_{1}\right\rangle \in \mathrm{EQ}\left(\Sigma_{1}\right)$. It must be shown that

$$
\begin{aligned}
\operatorname{ALG}(k)\left(\left\langle\left\langle\operatorname{SEN}^{\Omega^{N}(T)}\left(\Sigma^{\prime}\right),\left\{\sigma_{\Sigma^{\prime}}^{\Omega^{N}(T)}\right\}\right\rangle, f\right\rangle\right) \models \Sigma_{1}\left\langle t_{0}, t_{1}\right\rangle \quad \text { iff } \\
\left\langle\left\langle\operatorname{SEN}^{\Omega^{N}(T)}\left(\Sigma^{\prime}\right),\left\{\sigma_{\Sigma^{\prime}}^{\Omega^{N}(T)}\right\}\right\rangle, f\right\rangle \models \Sigma_{2} \operatorname{EQ}(k)\left(\left\langle t_{0}, t_{1}\right\rangle\right) .
\end{aligned}
$$

This is equivalent to the condition

$$
\begin{aligned}
\left\langle\left\langle\operatorname{SEN}^{\Omega^{N}(T)}\left(\Sigma^{\prime}\right),\left\{\sigma_{\Sigma^{\prime}}^{\Omega^{N}(T)}\right\}\right\rangle, f k\right\rangle \models \Sigma_{1}\left\langle t_{0}, t_{1}\right\rangle \quad \text { iff } \\
\\
\left\langle\left\langle\operatorname{SEN}^{\Omega^{N}(T)}\left(\Sigma^{\prime}\right),\left\{\sigma_{\Sigma^{\prime}}^{\Omega^{N}(T)}\right\}\right\rangle, f\right\rangle \models \Sigma_{2}\left\langle k^{*}\left(t_{0}\right), k^{*}\left(t_{1}\right)\right\rangle,
\end{aligned}
$$

which, in turn, is equivalent to

$$
\begin{aligned}
\operatorname{Eval}^{\left\langle N, T, \Sigma^{\prime}\right\rangle}\left((f k)^{*}\left(t_{0}\right)\right) & =\operatorname{Eval}^{\left\langle N, T, \Sigma^{\prime}\right\rangle}\left((f k)^{*}\left(t_{1}\right)\right) \quad \text { iff } \\
& \operatorname{Eval}^{\left\langle N, T, \Sigma^{\prime}\right\rangle}\left(f^{*}\left(k^{*}\left(\left(t_{0}\right)\right)\right)=\operatorname{Eval}^{\left\langle N, T, \Sigma^{\prime}\right\rangle}\left(f^{*}\left(k^{*}\left(\left(t_{1}\right)\right)\right) .\right.\right.
\end{aligned}
$$

But this last equivalence is the content of Lemma 6.1. 
The institution $\mathscr{L}^{N}(\ell)$ will be called the $N$-pseudo-algebraic counterpart or the $N$ Lindenbaum-Tarski counterpart of the $\pi$-institution $\ell$. Algebraic institutions were introduced in [27]. The term pseudo-algebraic counterpart for the institution $\mathscr{L}^{N}(\ell)$ is chosen because of its similarity with algebraic institutions. However, it should be noted that $\mathcal{L}^{N}(\ell)$ may not be genuinely algebraic, since the category Sign is an arbitrary category and not necessarily a full subcategory of a Kleisli category of some nontrivial algebraic theory (one of the requirements for an algebraic institution in [27]).

It is now shown that, given a $\pi$-institution $\ell=\langle\operatorname{Sign}, \mathrm{SEN}, C\rangle$, with $N$ a category of natural transformations on $\operatorname{SEN}, \Sigma_{0} \in|\operatorname{Sign}|$, and $\varphi, \psi \in \operatorname{SEN}\left(\Sigma_{0}\right)$, there exists a least theory system $T^{\left\langle\Sigma_{0}, \varphi, \psi\right\rangle}=\left\{T_{\Sigma}^{\left\langle\Sigma_{0}, \varphi, \psi\right\rangle}\right\}_{\Sigma \in \mid \text { Sign| }}$ such that $\langle\varphi, \psi\rangle \in \Omega_{\Sigma_{0}}^{N}\left(T^{\left\langle\Sigma_{0}, \varphi, \psi\right\rangle}\right)$. This theory system will enable the construction of a mapping (unfortunately not a translation) from the $N$-Lindenbaum-Tarski counterpart $\mathcal{L}^{N}(\ell)$ of $\ell$ into $\ell$ playing, roughly speaking, the role that the collection $E_{C}$ of formulas plays in the context of deductive systems in the proof of Theorem 1.1.3 of $[8]$ and subsequent related results.

Lemma 6.3 Let $\ell=\langle$ Sign, SEN, $C\rangle$ be a $\pi$-institution, $N$ a category of natural transformations on SEN, $\Sigma_{0} \in|\operatorname{Sign}|$, and $\varphi, \psi \in \operatorname{SEN}\left(\Sigma_{0}\right)$. Then there exists a least theory system $T^{\left\langle\Sigma_{0}, \varphi, \psi\right\rangle}=\left\{T_{\Sigma}^{\left\langle\Sigma_{0}, \varphi, \psi\right\rangle}\right\}_{\Sigma \in|\mathbf{S i g n}|}$ of $\ell$ such that $\langle\varphi, \psi\rangle \in \Omega_{\Sigma_{0}}^{N}\left(T^{\left\langle\Sigma_{0}, \varphi, \psi\right\rangle}\right)$.

Proof Let $\mathcal{T}$ be the collection of all theory systems $T$ of $\ell$ such that $\langle\varphi, \psi\rangle$ $\in \Omega_{\Sigma_{0}}^{N}(T)$. Clearly the largest theory system $\{\operatorname{SEN}(\Sigma)\}_{\Sigma \in|\operatorname{Sign}|} \in \mathcal{T}$. Also $\mathcal{T}$ is closed under signature-wise intersections. To see this, suppose that $T^{i} \in \mathcal{T}$, for all $i \in I$. Then $\langle\varphi, \psi\rangle \in \Omega_{\Sigma_{0}}^{N}\left(T^{i}\right)$, for all $i \in I$. This means, by Proposition 2.4 of [24], that, for all $\Sigma \in|\operatorname{Sign}|, f \in \operatorname{Sign}\left(\Sigma_{0}, \Sigma\right), \sigma: \operatorname{SEN}^{k} \rightarrow \operatorname{SEN}$ in $N$, and $\vec{\chi} \in \operatorname{SEN}(\Sigma)^{k-1}$,

$$
\sigma_{\Sigma}(\operatorname{SEN}(f)(\varphi), \vec{\chi}) \in T_{\Sigma}^{i} \quad \text { iff } \quad \sigma_{\Sigma}(\operatorname{SEN}(f)(\psi), \vec{\chi}) \in T_{\Sigma}^{i}, \quad i \in I .
$$

Thus, we immediately obtain that, for all $\Sigma \in|\operatorname{Sign}|, f \in \operatorname{Sign}\left(\Sigma_{0}, \Sigma\right), \sigma: \operatorname{SEN}^{k}$ $\rightarrow \mathrm{SEN}$ in $N$, and $\vec{\chi} \in \mathrm{SEN}(\Sigma)^{k-1}$,

$$
\sigma_{\Sigma}(\operatorname{SEN}(f)(\varphi), \vec{\chi}) \in \bigcap_{i \in I} T_{\Sigma}^{i} \quad \text { iff } \quad \sigma_{\Sigma}(\operatorname{SEN}(f)(\psi), \vec{\chi}) \in \bigcap_{i \in I} T_{\Sigma}^{i},
$$

which, again, by Proposition 2.4 of [24], yields that $\langle\varphi, \psi\rangle \in \Omega_{\Sigma_{0}}^{N}\left(\bigcap_{i \in I} T^{i}\right)$. Therefore, the theory system $\bigcap \mathcal{T}$ is the least theory system of $\ell$ such that $\langle\varphi, \psi\rangle \in \Omega_{\Sigma}^{N}(\bigcap \mathcal{T})$

Sometimes the notation $T\left(\Sigma_{0}, \varphi, \psi\right)$ will be used instead of the notation $T^{\left\langle\Sigma_{0}, \varphi, \psi\right\rangle}$ for typographical purposes.

Next, given a $\pi$-institution $\ell=\langle$ Sign, SEN, $C\rangle$, with $N$ a category of natural transformations on SEN, let $\left\langle\mathrm{I}_{\text {Sign }}, \beta\right\rangle: \mathscr{L}^{N}(\ell) \rightarrow \ell$ be defined, for all $\Sigma \in|\mathbf{S i g n}|,\left\langle t_{0}, t_{1}\right\rangle \in \mathrm{EQ}(\Sigma)$, by

$$
\beta_{\Sigma}\left(\left\langle t_{0}, t_{1}\right\rangle\right)=T_{\Sigma}\left(\Sigma, \operatorname{Eval}^{\left\langle\operatorname{SEN}(\Sigma),\left\{\sigma_{\Sigma}\right\}\right\rangle}\left(t_{0}\right), \operatorname{Eval}^{\left\langle\operatorname{SEN}(\Sigma),\left\{\sigma_{\Sigma}\right\}\right\rangle}\left(t_{0}\right)\right),
$$

where, as before, $\operatorname{Eval}^{\left\langle\operatorname{SEN}(\Sigma),\left\{\sigma_{\Sigma}\right\}\right\rangle}: \operatorname{Tm}_{N^{\#}}(\operatorname{SEN}(\Sigma)) \rightarrow \operatorname{SEN}(\Sigma)$ is the function that evaluates $N^{\#}$-terms into the $N^{\#}$-algebra $\left\langle\operatorname{SEN}(\Sigma),\left\{\sigma_{\Sigma}\right\}\right\rangle$ under the identity interpretation. 
It would have been a very satisfactory state of affairs from the point of view of [27] and [28] if it had been possible to show that $\left\langle\mathrm{I}_{\operatorname{Sign}}, \beta\right\rangle: \mathcal{L}^{N}(\ell) \rightarrow \ell$ is a translation; that is, if, for all $\Sigma_{1}, \Sigma_{2} \in|\operatorname{Sign}|, f \in \operatorname{Sign}\left(\Sigma_{1}, \Sigma_{2}\right)$, we had that

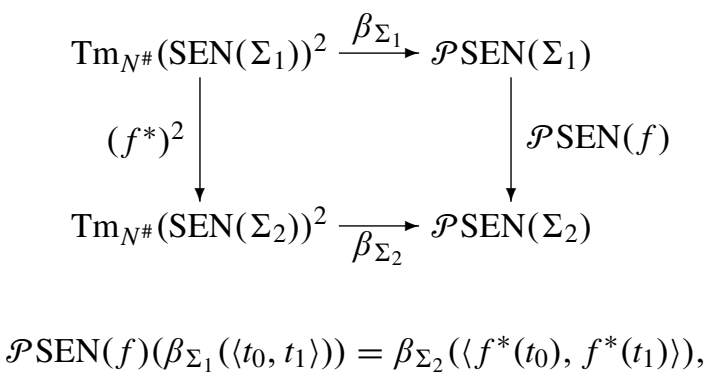

for all $\left\langle t_{0}, t_{1}\right\rangle \in \operatorname{Tm}_{N^{*}}\left(\operatorname{SEN}\left(\Sigma_{1}\right)\right)^{2}$. Of course this equation is unreasonably ambitious because, on the one hand, $\beta_{\Sigma_{2}}\left(\left\langle f^{*}\left(t_{0}\right), f^{*}\left(t_{1}\right)\right\rangle\right)$ is a $\Sigma_{2}$-theory whereas $\mathcal{P S E N}(f)\left(\beta_{\Sigma_{1}}\left(\left\langle t_{0}, t_{1}\right\rangle\right)\right)$ does not have to be a $\Sigma_{2}$-theory. Since Equation (7) is too strong to hold in general, we will settle with establishing two lemmas about properties that can be demonstrated to hold in general about $\beta$. First, it will be shown that the evaluation function commutes with the sentence functor; that is, it is natural. Second, it will be shown that both $\beta_{\Sigma_{2}}\left(\left\langle f^{*}\left(t_{0}\right), f^{*}\left(t_{1}\right)\right\rangle\right)$ and $\mathcal{P S E N}(f)\left(\beta_{\Sigma_{1}}\left(\left\langle t_{0}, t_{1}\right\rangle\right)\right)$ have as a common upper bound under inclusion the $\Sigma_{2}$-theory

$$
T_{\Sigma_{2}}\left(\Sigma_{1}, \operatorname{Eval}^{\left\langle\operatorname{SEN}\left(\Sigma_{1}\right),\left\{\sigma_{\Sigma_{1}}\right\}\right\rangle}\left(t_{0}\right), \mathrm{Eval}^{\left\langle\operatorname{SEN}\left(\Sigma_{1}\right),\left\{\sigma_{\Sigma_{1}}\right\}\right\rangle}\left(t_{0}\right)\right)
$$

of $\ell$.

Lemma 6.4 Let $\ell=\langle$ Sign, SEN, $C\rangle$ be a $\pi$-institution and $N$-a category of natural transformations on SEN. Then, for every $\Sigma_{1}, \Sigma_{2} \in|\operatorname{Sign}|, f \in \operatorname{Sign}\left(\Sigma_{1}, \Sigma_{2}\right)$, and all $t \in \operatorname{Tm}_{N^{\#}}\left(\operatorname{SEN}\left(\Sigma_{1}\right)\right)$,

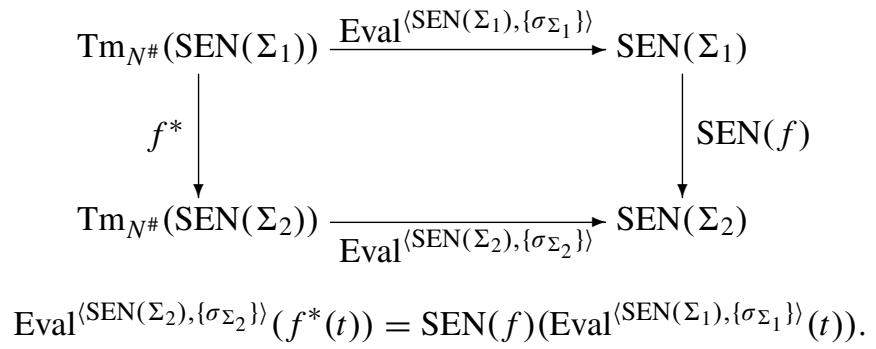

Proof To show the required commutativity we work by induction on the structure of $N^{\#}$-terms over $\operatorname{SEN}\left(\Sigma_{1}\right)$. For the base case, if $\varphi \in \operatorname{SEN}\left(\Sigma_{1}\right)$, then $f^{*}(\varphi)=\operatorname{SEN}(f)(\varphi)$, whence

$$
\begin{aligned}
\operatorname{Eval}^{\left\langle\operatorname{SEN}\left(\Sigma_{2}\right),\left\{\sigma_{\Sigma_{2}}\right\}\right\rangle}\left(f^{*}(\varphi)\right) & =\operatorname{Eval}^{\left\langle\operatorname{SEN}\left(\Sigma_{2}\right),\left\{\sigma_{\Sigma_{2}}\right\}\right\rangle}(\operatorname{SEN}(f)(\varphi)) \\
& =\operatorname{SEN}(f)(\varphi) \\
& =\operatorname{SEN}(f)\left(\operatorname{Eval}{ }^{\left\langle\operatorname{SEN}\left(\Sigma_{1}\right),\left\{\sigma_{\Sigma_{1}}\right\}\right\rangle}(\varphi)\right) .
\end{aligned}
$$

For the induction step, suppose that $t=\sigma^{\#}\left(t_{0}, \ldots, t_{n-1}\right)$ and that, according to the induction hypothesis, for all $i=0,1, \ldots, n-1$,

$$
\operatorname{Eval}^{\left\langle\operatorname{SEN}\left(\Sigma_{2}\right),\left\{\sigma_{\Sigma_{2}}\right\}\right\rangle}\left(f^{*}\left(t_{i}\right)\right)=\operatorname{SEN}(f)\left(\operatorname{Eval}{ }^{\left\langle\operatorname{SEN}\left(\Sigma_{1}\right),\left\{\sigma_{\Sigma_{1}}\right\}\right\rangle}\left(t_{i}\right)\right) .
$$


Then we get

$$
\begin{aligned}
& \operatorname{Eval}^{\left\langle\operatorname{SEN}\left(\Sigma_{2}\right),\left\{\sigma_{\Sigma_{2}}\right\}\right\rangle}\left(f^{*}\left(\sigma^{\#}\left(t_{0}, \ldots, t_{n-1}\right)\right)\right)= \\
& =\operatorname{Eval}^{\left\langle\operatorname{SEN}\left(\Sigma_{2}\right),\left\{\sigma_{\Sigma_{2}}\right\}\right\rangle}\left(\sigma^{\#}\left(f^{*}\left(t_{0}\right), \ldots, f^{*}\left(t_{n-1}\right)\right)\right) \\
& =\sigma\left(\operatorname{Eval}{ }^{\left\langle\operatorname{SEN}\left(\Sigma_{2}\right),\left\{\sigma_{\Sigma_{2}}\right\}\right\rangle}\left(f^{*}\left(t_{0}\right)\right), \ldots,\left.\operatorname{Eval}\right|^{\left.\operatorname{SEN}\left(\Sigma_{2}\right),\left\{\sigma_{\Sigma_{2}}\right\}\right\rangle}\left(f^{*}\left(t_{n-1}\right)\right)\right) \\
& =\sigma\left(\operatorname{SEN}(f)\left(\operatorname{Eval}^{\left\langle\operatorname{SEN}\left(\Sigma_{1}\right),\left\{\sigma_{\Sigma_{1}}\right\}\right\rangle}\left(t_{0}\right)\right), \ldots,\right. \\
& \left.\operatorname{SEN}(f)\left(\operatorname{Eval}{ }^{\left\langle\operatorname{SEN}\left(\Sigma_{1}\right),\left\{\sigma_{\Sigma_{1}}\right\}\right\rangle}\left(t_{n-1}\right)\right)\right) \\
& =\operatorname{SEN}(f)\left(\sigma\left(\operatorname{Eval}^{\left\langle\operatorname{SEN}\left(\Sigma_{1}\right),\left\{\sigma_{\Sigma_{1}}\right\}\right\rangle}\left(t_{0}\right), \ldots, \operatorname{Eval}^{\left\langle\operatorname{SEN}\left(\Sigma_{1}\right),\left\{\sigma_{\Sigma_{1}}\right\}\right\rangle}\left(t_{n-1}\right)\right)\right) \\
& =\operatorname{SEN}(f)\left(\operatorname{Eval}{ }^{\left\langle\operatorname{SEN}\left(\Sigma_{1}\right),\left\{\sigma_{\Sigma_{1}}\right\}\right\rangle}\left(\sigma^{\#}\left(t_{0}, \ldots, t_{n-1}\right)\right)\right) \text {. }
\end{aligned}
$$

Taking into account the result of Lemma 6.4, the ideal Equation (7) would have taken the form

$$
\begin{aligned}
& \mathcal{P S E N}(f)\left(T_{\Sigma_{1}}\left(\Sigma_{1}, \operatorname{Eval}^{\left\langle\operatorname{SEN}\left(\Sigma_{1}\right),\left\{\sigma_{\Sigma_{1}}\right\}\right\rangle}\left(t_{0}\right), \operatorname{Eval}^{\left\langle\operatorname{SEN}\left(\Sigma_{1}\right),\left\{\sigma_{\Sigma_{1}}\right\}\right\rangle}\left(t_{1}\right)\right)\right)= \\
& T_{\Sigma_{2}}\left(\Sigma_{2}, \operatorname{SEN}(f)\left(\operatorname{Eval}^{\left\langle\operatorname{SEN}\left(\Sigma_{1}\right),\left\{\sigma_{\Sigma_{1}}\right\}\right\rangle}\left(t_{0}\right)\right), \operatorname{SEN}(f)\left(\operatorname{Eval}^{\left\langle\operatorname{SEN}\left(\Sigma_{1}\right),\left\{\sigma_{\Sigma_{1}}\right\}\right\rangle}\left(t_{1}\right)\right)\right),
\end{aligned}
$$

for all $\left\langle t_{0}, t_{1}\right\rangle \in \operatorname{Tm}_{N^{\#}}\left(\operatorname{SEN}\left(\Sigma_{1}\right)\right)^{2}$. Or, denoting by

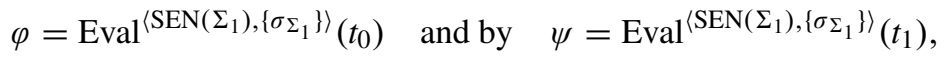

$$
\begin{aligned}
& \mathcal{P S E N}(f)\left(T_{\Sigma_{1}}\left(\Sigma_{1}, \varphi, \psi\right)\right)=T_{\Sigma_{2}}\left(\Sigma_{2}, \operatorname{SEN}(f)(\varphi), \operatorname{SEN}(f)(\psi)\right),
\end{aligned}
$$

for all $\langle\varphi, \psi\rangle \in \operatorname{SEN}\left(\Sigma_{1}\right)^{2}$; that is, the essence of the part of Equation (7) that does not hold revolves around a failing commutativity property between signature morphisms and the construction of the minimal theory of Lemma 6.3. The best that we can do in this direction, for now, is establish that both sides of the failing Equation (8) are included in the same $\Sigma_{2}$-theory of $\ell$.

Lemma 6.5 Let $\ell=\langle$ Sign, SEN, $C\rangle$ be a $\pi$-institution with $N$ a category of natural transformations on $\mathrm{SEN}$. Then, for all $\Sigma_{1}, \Sigma_{2} \in|\operatorname{Sign}|, f \in \operatorname{Sign}\left(\Sigma_{1}, \Sigma_{2}\right)$, and all $\varphi, \psi \in \operatorname{SEN}\left(\Sigma_{1}\right)$,

$$
\begin{aligned}
& \operatorname{PSEN}(f)\left(T_{\Sigma_{1}}\left(\Sigma_{1}, \varphi, \psi\right)\right) \subseteq T_{\Sigma_{2}}\left(\Sigma_{1}, \varphi, \psi\right) \text { and } \\
& T_{\Sigma_{2}}\left(\Sigma_{2}, \operatorname{SEN}(f)(\varphi), \operatorname{SEN}(f)(\psi)\right) \subseteq T_{\Sigma_{2}}\left(\Sigma_{1}, \varphi, \psi\right) .
\end{aligned}
$$

Proof The first inclusion is obvious since $T\left(\Sigma_{1}, \varphi, \psi\right)$ is, by its definition, a theory system of $\ell$ and, by the definition of a theory system, if $\chi \in T_{\Sigma_{1}}\left(\Sigma_{1}, \varphi, \psi\right)$, then $\operatorname{SEN}(f)(\chi) \in T_{\Sigma_{2}}\left(\Sigma_{1}, \varphi, \psi\right)$. The second inclusion uses the minimality of Lemma 6.3. Note that $T\left(\Sigma_{1}, \varphi, \psi\right)$ is, by Lemma 6.3, a theory system such that $\langle\varphi, \psi\rangle \in \Omega_{\Sigma_{1}}^{N}\left(T\left(\Sigma_{1}, \varphi, \psi\right)\right)$. But $\Omega^{N}\left(T\left(\Sigma_{1}, \varphi, \psi\right)\right)$ is by definition an $N$-congruence system, whence we get that $\langle\operatorname{SEN}(f)(\varphi), \operatorname{SEN}(f)(\psi)\rangle$ $\in \Omega_{\Sigma_{2}}^{N}\left(T\left(\Sigma_{1}, \varphi, \psi\right)\right)$. But, by Lemma 6.3, $T\left(\Sigma_{2}, \operatorname{SEN}(f)(\varphi), \operatorname{SEN}(f)(\psi)\right)$ is the least theory system $T^{\prime}$ of $\ell$ such that $\langle\operatorname{SEN}(f)(\varphi), \operatorname{SEN}(f)(\psi)\rangle \in \Omega_{\Sigma_{2}}^{N}\left(T^{\prime}\right)$. Therefore, we obtain that

$$
T\left(\Sigma_{2}, \operatorname{SEN}(f)(\varphi), \operatorname{SEN}(f)(\psi)\right) \leq T\left(\Sigma_{1}, \varphi, \psi\right),
$$

which, specialized over $\Sigma_{2}$, yields the second inclusion. 
The mapping $\left\langle\mathrm{I}_{\text {Sign }}, \beta\right\rangle: \mathcal{L}^{N}(\ell) \rightarrow \ell$ (it is denoted like a translation by abusing notation) will be called the $N$-Blok-Pigozzi mapping of $\ell$. Note that, roughly speaking, it gives an "automatic" way to pass from equations of the equational-like Lindenbaum-Tarski counterpart of an arbitrary $\pi$-institution $\ell$ to collections of sentences (actually theories) of $\ell$.

In the case of deductive systems, as studied by Blok and Pigozzi, this mapping always exists and, in addition, is always a translation. This happens because the restricted framework of deductive systems is endowed with many additional features that are not present in general in an arbitrary $\pi$-institution. One basic feature is that the logical connectives of a deductive system coincide with the operation symbols of its algebraic models. As a result, the formulas of a deductive system are identical with the terms of the free algebra, built with operation symbols the connectives of the deductive system. An even more fundamental difference is the existence, in the universal algebraic framework of deductive systems, of a free algebra that is built up of sentential variables. When theories come into play, one is always able to choose generators and manipulate the variables appearing in the generators instead of having to carry the entire theories along. This phenomenon is unfortunately not paralleled in an arbitrary $\pi$-institution.

Next it is shown that for an arbitrary $\pi$-institution $\ell=\langle$ Sign, SEN, $C\rangle$ with $N$ a category of natural transformations on SEN, the $N$-Blok-Pigozzi mapping $\left\langle\mathrm{I}_{\text {Sign }}, \beta\right\rangle: \mathscr{L}^{N}(\ell) \rightarrow \ell$, from its $N$-Lindenbaum-Tarski counterpart $\mathcal{L}^{N}(\ell)$ into itself, always behaves like a semi-interpretation.

Proposition 6.6 Let $\ell=\langle\mathrm{Sign}, \mathrm{SEN}, C\rangle$ be a $\pi$-institution with $N$ a category of natural transformations on SEN. Then the Blok-Pigozzi mapping $\left\langle\mathrm{I}_{\mathbf{S i g n}}, \beta\right\rangle$ : $\mathcal{L}^{N}(\ell) \rightarrow \ell$ satisfies

$$
E \models_{\Sigma}\left\langle t_{0}, t_{1}\right\rangle \text { implies } \beta_{\Sigma}\left(\left\langle t_{0}, t_{1}\right\rangle\right) \subseteq C_{\Sigma}\left(\beta_{\Sigma}(E)\right),
$$

for all $\Sigma \in|\mathbf{S i g n}|, E \cup\left\{\left\langle t_{0}, t_{1}\right\rangle\right\} \subseteq \mathrm{EQ}(\Sigma)$.

Proof We need to prove that, for all $\Sigma \in|\mathbf{S i g n}|$ and all $E \cup\left\{\left\langle t_{0}, t_{1}\right\rangle\right\} \subseteq$ $\operatorname{Tm}_{N^{*}}(\operatorname{SEN}(\Sigma))^{2}$,

$$
E \models_{\Sigma}\left\langle t_{0}, t_{1}\right\rangle \text { implies } \quad \beta_{\Sigma}\left(\left\langle t_{0}, t_{1}\right\rangle\right) \subseteq C_{\Sigma}\left(\beta_{\Sigma}(E)\right) .
$$

If $E \models \Sigma\left\langle t_{0}, t_{1}\right\rangle$, then, for all $\Sigma^{\prime} \in|\operatorname{Sign}|, f \in \operatorname{Sign}\left(\Sigma, \Sigma^{\prime}\right), T$ a theory family of $\ell$, we have that, if $\operatorname{Eval}^{\left\langle N, T, \Sigma^{\prime}\right\rangle}\left(f^{*}\left(e_{0}\right)\right)=\operatorname{Eval}^{\left\langle N, T, \Sigma^{\prime}\right\rangle}\left(f^{*}\left(e_{1}\right)\right)$, for all $\left\langle e_{0}, e_{1}\right\rangle \in E$, then $\operatorname{Eval}^{\left\langle N, T, \Sigma^{\prime}\right\rangle}\left(f^{*}\left(t_{0}\right)\right)=\operatorname{Eval}^{\left\langle N, T, \Sigma^{\prime}\right\rangle}\left(f^{*}\left(t_{1}\right)\right)$. Taking $f=i_{\Sigma}$, the identity on $\Sigma$, and $T=T\left(\Sigma, \operatorname{Eval}^{\left\langle\operatorname{SEN}(\Sigma),\left\{\sigma_{\Sigma}\right\}\right\rangle}(E)\right)$, and, since

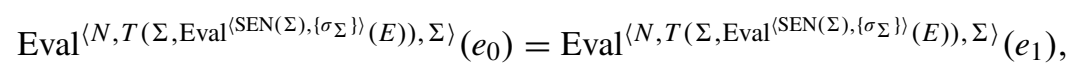

for all $\left\langle e_{0}, e_{1}\right\rangle \in E$, we get that

$$
\operatorname{Eval}^{\left\langle N, T\left(\Sigma, \operatorname{Eval}^{\left\langle\operatorname{SEN}(\Sigma),\left\{\sigma_{\Sigma}\right\rangle\right\rangle}(E)\right), \Sigma\right\rangle}\left(t_{0}\right)=\operatorname{Eval}^{\left\langle N, T\left(\Sigma, \operatorname{Eval}^{\left\langle\operatorname{SEN}(\Sigma),\left\{\sigma_{\Sigma}\right\}\right\rangle}(E)\right), \Sigma\right\rangle}\left(t_{1}\right) ;
$$

that is,

$$
\begin{aligned}
\left\langle\operatorname{Eval}^{\left\langle\operatorname{SEN}(\Sigma),\left\{\sigma_{\Sigma}\right\}\right\rangle}\left(t_{0}\right), \mathrm{Eval}^{\left\langle\operatorname{SEN}(\Sigma),\left\{\sigma_{\Sigma}\right\}\right\rangle}\left(t_{1}\right)\right\rangle & \in \\
& \Omega_{\Sigma}^{N}\left(T\left(\Sigma, \operatorname{Eval}^{\left\langle\operatorname{SEN}(\Sigma),\left\{\sigma_{\Sigma}\right\}\right\rangle}(E)\right)\right) .
\end{aligned}
$$


Therefore, by the minimality property of Lemma 6.3 , we get that

$$
\begin{aligned}
T_{\Sigma}\left(\Sigma, \mathrm{Eval}^{\left\langle\operatorname{SEN}^{N}(\Sigma), \sigma_{\Sigma}^{N}\right\rangle}\left(t_{0}\right), \mathrm{Eval}^{\left\langle\operatorname{SEN}^{N}(\Sigma), \sigma_{\Sigma}^{N}\right\rangle}\left(t_{1}\right)\right) \subseteq & \\
& T_{\Sigma}\left(\Sigma, \mathrm{Eval}^{\left\langle\operatorname{SEN}^{N}(\Sigma), \sigma_{\Sigma}^{N}\right\rangle}(E)\right) .
\end{aligned}
$$

But this is exactly the condition in the conclusion of (9).

Given a $\pi$-institution $\ell=\langle$ Sign, SEN, $C\rangle$ with $N$ a category of natural transformations on SEN and $P$ a collection of natural transformations $p: \mathrm{SEN}^{2} \rightarrow$ SEN in $N, \ell$ will be said to have the $(\beta, N)$-translation property with respect to $P$ if, for all $\Sigma \in|\operatorname{Sign}|$ and all $\varphi, \psi \in \operatorname{SEN}(\Sigma)$,

$$
T_{\Sigma}(\Sigma, \varphi, \psi)=C_{\Sigma}\left(P_{\Sigma}(\varphi, \psi)\right),
$$

where, as usual, $P_{\Sigma}(\varphi, \psi)=\left\{p_{\Sigma}(\varphi, \psi): p \in P\right\}$.

The discussion preceding and leading up to Equation (8) shows that Proposition 6.6 has the following corollary.

Corollary 6.7 Let $\ell=\langle$ Sign, SEN, $C\rangle$ be a $\pi$-institution with $N$ a category of natural transformations on $\mathrm{SEN}$. If $\ell$ has the $(\beta, N)$-translation property with respect to a collection $P$, then $\left\langle\mathrm{I}_{\text {Sign }}, P\right\rangle: \mathcal{L}^{N}(\ell) \rightarrow \ell$ is a semi-interpretation and

$$
\beta_{\Sigma}\left(\left\langle t_{0}, t_{1}\right)\right\rangle=C_{\Sigma}\left(P_{\Sigma}\left(\operatorname{Eval}^{\left\langle\operatorname{SEN}(\Sigma),\left\{\sigma_{\Sigma}\right\}\right\rangle}\left(t_{0}\right), \operatorname{Eval}^{\left\langle\operatorname{SEN}(\Sigma),\left\{\sigma_{\Sigma}\right\}\right\rangle}\left(t_{0}\right)\right)\right),
$$

for all $\Sigma \in|\mathbf{S i g n}|, t_{0}, t_{1} \in \mathrm{EQ}(\Sigma)$.

Finally, it is shown that the Blok-Pigozzi mapping has a modus ponens-like property in an $N$-protoalgebraic $\pi$-institution $\ell$.

Proposition 6.8 Let $\ell=\langle$ Sign, SEN, $C\rangle$ be a $\pi$-institution with $N$ a category of natural transformations on SEN. If $\ell$ is $N$-protoalgebraic, then, for every $\Sigma \in|\mathbf{S i g n}|$ and all $\varphi, \psi \in \operatorname{SEN}(\Sigma)$

$$
\psi \in C_{\Sigma}\left(T_{\Sigma}(\Sigma, \varphi, \psi) \cup\{\varphi\}\right) .
$$

Proof To show the required inclusion, suppose that $T$ is an arbitrary theory family of $\ell$ such that $T(\Sigma, \varphi, \psi) \leq T$ and $\varphi \in T_{\Sigma}$. Then since $T(\Sigma, \varphi, \psi) \leq T$, we obtain, by $N$-protoalgebraicity, that $\Omega^{N}(T(\Sigma, \varphi, \psi)) \leq \Omega^{N}(T)$, whence, since, by the definition of $T(\Sigma, \varphi, \psi),\langle\varphi, \psi\rangle \in \Omega_{\Sigma}^{N}(T(\Sigma, \varphi, \psi))$, we get that $\langle\varphi, \psi\rangle \in \Omega_{\Sigma}^{N}(T)$. Thus since by hypothesis $\varphi \in T_{\Sigma}$, we obtain by the compatibility property of $\Omega^{N}(T)$ with respect to $T$ that $\psi \in T_{\Sigma}$. Therefore, since $T$ was arbitrary with $T(\Sigma, \varphi, \psi) \leq T$ and $\varphi \in T_{\Sigma}$, we get $\psi \in C_{\Sigma}\left(T_{\Sigma}(\Sigma, \varphi, \psi) \cup\{\varphi\}\right)$.

Proposition 6.8 yields Corollary 6.9 which is a converse to Proposition 3.2 in the special case when $\ell$ has the $(\beta, N)$-translation property.

Corollary 6.9 Let $\ell=\langle$ Sign, SEN, $C\rangle$ be a $\pi$-institution with $N$ a category of natural transformations on $\mathrm{SEN}$, and suppose that $\ell$ has the $(\beta, N)$-translation property with respect to a collection $P$. Then $\ell$ is $N$-protoalgebraic if and only if, for every $\Sigma \in|\operatorname{Sign}|$ and all $\varphi, \psi \in \operatorname{SEN}(\Sigma)$,

$$
\psi \in C_{\Sigma}\left(P_{\Sigma}(\varphi, \psi) \cup\{\varphi\}\right) .
$$




\section{References}

[1] Barr, M., and C. Wells, Category Theory for Computing Science, 3d edition, Les Publications CRM, Montreal, 1999. Zbl 0714.18001. MR 1094561. 490

[2] Blok, W. J., and D. Pigozzi, "Algebraic semantics for universal Horn logic without equality," pp. 1-56 in Universal Algebra and Quasi-group Theory (Jadwisin, 1989), edited by A. Romanowska and J. D. H. Smith, vol. 19 of Research and Exposition in Mathematics, Heldermann, Berlin, 1992. Zbl 0768.03008. MR 1191226. 489

[3] Blok, W. J., and D. Pigozzi, "Protoalgebraic logics," Studia Logica, vol. 45 (1986), pp. 337-69. Zbl 0622.03020. MR 884144. 487, 488, 492, 493

[4] Blok, W. J., and D. Pigozzi, "Algebraizable logics," Memoirs of the American Mathematical Society, vol. 77 (1989), no. 396. Zbl 0664.03042. MR 973361. 488, 489, 495

[5] Borceux, F., Handbook of Categorical Algebra. Vol. 1. Basic Category Theory, vol. 50 of Encyclopedia of Mathematics and its Applications, Cambridge University Press, Cambridge, 1994. Zbl 0803.18001. MR 1291599. 490

[6] Czelakowski, J., "Equivalential logics. I and II," Studia Logica, vol. 40 (1981), pp. 22736 and 355-72. Zbl 0476.03032. MR 665717. 488, 489

[7] Czelakowski, J., "Filter distributive logics," Studia Logica, vol. 43 (1984), pp. 353-77. Zbl 0578.03012. MR 803314. 488

[8] Czelakowski, J., Protoalgebraic Logics, vol. 10 of Trends in Logic-Studia Logica Library, Kluwer Academic Publishers, Dordrecht, 2001. Zbl 0984.03002. MR 1828895. 487, 488, 489, 492, 494, 495, 496, 497, 498, 507

[9] Czelakowski, J., and W. Dziobiak, "A deduction theorem schema for deductive systems of propositional logics," Studia Logica, vol. 50 (1991), pp. 385-90. Special Issue on Algebraic Logic. Zbl 0755.03014. MR 1170181. 493

[10] Czelakowski, J., and R. Jansana, "Weakly algebraizable logics," The Journal of Symbolic Logic, vol. 65 (2000), pp. 641-68. Zbl 0960.03055. MR 1771075. 489

[11] Fiadeiro, J., and A. Sernadas, "Structuring theories on consequence," pp. 44-72 in Recent Trends in Data Type Specification, edited by D. Sannella and A. Tarlecki, vol. 332 of Lecture Notes in Computer Science, Springer-Verlag, New York, 1988. Papers from the Fifth Workshop on Specification of Abstract Data Types held in Gullane, September 1-4, 1987. Zbl 0671.03020. MR 1031720. 488

[12] Font, J. M., R. Jansana, and D. Pigozzi, "A survey of abstract algebraic logic," Studia Logica, vol. 74 (2003), pp. 13-97. Abstract Algebraic Logic, Part II (Barcelona, 1997). Zbl 1057.03058. MR 1996593. 487, 488

[13] Font, J. M., and R. Jansana, A General Algebraic Semantics for Sentential Logics, vol. 7 of Lecture Notes in Logic, Springer-Verlag, Berlin, 1996. Zbl 0865.03054. MR 1421569. $488,489,499,500,501,503$

[14] Goguen, J. A., and R. M. Burstall, "Introducing institutions," pp. 221-56 in Proceedings of the Logic of Programming Workshop (Pittsburgh, 1983), edited by E. Clarke and D. Kozen, vol. 164 of Lecture Notes in Computer Science, Springer-Verlag, New York, 1984. Zbl 0543.68021. MR 778942. 488 
[15] Goguen, J. A., and R. M. Burstall, "Institutions: Abstract model theory for specification and programming," Journal of the Association for Computing Machinery, vol. 39 (1992), pp. 95-146. Zbl 0799.68134. MR 1147298. 488

[16] Herrmann, B., Equivalential Logics and Definability of Truth, Ph.D. thesis, Freie Universitat Berlin, Berlin, 1993. 488

[17] Herrmann, B., "Equivalential and algebraizable logics," Studia Logica, vol. 57 (1996), pp. 419-36. Zbl 0864.03043. MR 1416744. 488

[18] Herrmann, B., "Characterizing equivalential and algebraizable logics by the Leibniz operator," Studia Logica, vol. 58 (1997), pp. 305-23. Zbl 0879.03023. MR 1439022. 488

[19] MacLane, S., Categories for the Working Mathematician, vol. 5 of Graduate Texts in Mathematics, Springer-Verlag, New York, 1971. Zbl 0705.18001. MR 0354798. 490

[20] Prucnal, T., and A. Wroński, "An algebraic characterization of the notion of structural completeness," Bulletin of the Section of Logic, vol. 3 (1974), pp. 30-33. MR 0403950. 488

[21] Voutsadakis, G., "Categorical Abstract Algebraic Logic: Full models, Frege systems and metalogical properties," Reports on Mathematical Logic, vol. 41 (2006), pp. 31-62. 489,491

[22] Voutsadakis, G., "Categorical Abstract Algebraic Logic: Generalized Tarski congruence systems," submitted to Theory and Applications of Categories. Preprint available at http://www.voutsadakis.com/RESEARCH/papers.html. 489

[23] Voutsadakis, G., "Categorical Abstract Algebraic Logic: Gentzen $\pi$-institutions," preprint available at http://www.voutsadakis.com/RESEARCH/papers.html. 489, 491

[24] Voutsadakis, G., "Categorical Abstract Algebraic Logic: Prealgebraicity and protoalgebraicity," forthcoming in Studia Logica. Preprint available at http://www.voutsadakis.com/RESEARCH/papers.html. 487, 488, 489, 490, 491, 492, 497, 499, 500, 501, 502, 507

[25] Voutsadakis, G., "Categorical Abstract Algebraic Logic: Tarski congruence systems, logical morphisms and logical quotients," submitted to the Annals of Pure and Applied Logic. Preprint available at http://www.voutsadakis.com/RESEARCH/papers.html. 489, 490, 491

[26] Voutsadakis, G., Categorical Abstract Algebraic Logic, Ph.D. thesis, Iowa State University, Ames, 1998. 489

[27] Voutsadakis, G., "Categorical Abstract Algebraic Logic: Algebraizable institutions," Applied Categorical Structures, vol. 10 (2002), pp. 531-68. Zbl 1028.03051. MR 1941391. 489, 507, 508

[28] Voutsadakis, G., "Categorical Abstract Algebraic Logic: Equivalent institutions,” Studia Logica, vol. 74 (2003), pp. 275-311. Abstract Algebraic Logic, Part II (Barcelona, 1997). Zbl 1043.03049. MR 1996598. 489, 508

[29] Voutsadakis, G., "Categorical Abstract Algebraic Logic: Metalogical properties," Studia Logica, vol. 74 (2003), pp. 369-98. Zbl 1030.03049. MR 1996835. 489 
[30] Voutsadakis, G., "Categorical Abstract Algebraic Logic: $(l, N)$-algebraic systems," Applied Categorical Structures, vol. 13 (2005), pp. 265-80. Zbl 1089.03057. MR 2167794. 489, 499, 500, 501, 503

[31] Voutsadakis, G., "Categorical Abstract Algebraic Logic: Models of $\pi$-institutions," Notre Dame Journal of Formal Logic, vol. 46 (2005), pp. 439-60. Zbl 1089.03058. MR 2183054. 489

\section{Acknowledgments}

The results presented in this paper would not have existed if the corresponding theory for deductive systems had not been developed to its present form. Many colleagues have to be acknowledged for this development, but the author would like to thank especially Don Pigozzi, Janusz Czelakowski, Josep Maria Font, and Ramon Jansana and to commemorate Wim Blok, whose works have had a deep influence on the work presented here and who have provided moral support and encouragement over many years. I would also like to extend warm thanks to Charles Wells and Giora Slutzki for their guidance and support.

School of Mathematics and Computer Science

Lake Superior State University

650 W Easterday Avenue

Sault Sainte Marie MI 49783

gvoutsad@Issu.edu

http://www.voutsadakis.com/main.html 